quatrième série-tome 42 fascicule 3 mai-juin 2009

$$
\begin{aligned}
& \text { ANNALES } \\
& \text { SCIENTIFIQUES } \\
& \text { de } \\
& \text { L'ECOLE } \\
& \text { NORMALE } \\
& \text { SUPÉRIEURE }
\end{aligned}
$$

Tommaso de FERNEX \& Mircea MUSTAȚĂ

Limits of log canonical thresholds 
Ann. Scient. Éc. Norm. Sup.

$4^{\mathrm{e}}$ série, t. 42, 2009, p. 491 à 515

\title{
LIMITS OF LOG CANONICAL THRESHOLDS
}

\author{
By Tommaso de FERnEX and Mircea MUSTAȚA
}

\begin{abstract}
Let $\mathcal{T}_{n}$ denote the set of $\log$ canonical thresholds of pairs $(X, Y)$, with $X$ a nonsingular variety of dimension $n$, and $Y$ a nonempty closed subscheme of $X$. Using non-standard methods, we show that every limit of a decreasing sequence in $\mathcal{T}_{n}$ lies in $\mathcal{T}_{n-1}$, proving in this setting a conjecture of Kollár. We also show that $\mathcal{T}_{n}$ is closed in $\mathbf{R}$; in particular, every limit of log canonical thresholds on smooth varieties of fixed dimension is a rational number. As a consequence of this property, we see that in order to check Shokurov's ACC Conjecture for all $\mathcal{T}_{n}$, it is enough to show that 1 is not a point of accumulation from below of any $\mathcal{T}_{n}$. In a different direction, we interpret the ACC Conjecture as a semi-continuity property for $\log$ canonical thresholds of formal power series.
\end{abstract}

RÉsumÉ. - Dans cet article, nous analysons les ensembles $\mathcal{T}_{n}$ de seuils $\log$ canoniques de paires $(X, Y)$, où $X$ est une variété lisse de dimension $n$, et $Y$ est un sous-schéma fermé non-vide de $X$. En employant des méthodes non-standard, nous montrons que chaque limite d'une suite strictement décroissante de $\mathcal{T}_{n}$ appartient à l'ensemble $\mathcal{T}_{n-1}$ (ce résultat a été conjecturé par J. Kollár dans ses travaux sur le sujet). Nous montrons également que l'ensemble $\mathcal{T}_{n}$ est fermé dans $\mathbf{R}$, et en déduisons que les valeurs adhérentes de l'ensemble des seuils log canoniques des pairs $(X, Y)$ sont rationnelles, si la dimension de $X$ est majorée. Une autre conséquence de nos résultats concerne la conjecture ACC de Shokurov pour les $\mathcal{T}_{n}$. En effet, nous montrons qu'elle est une conséquence de l'énoncé suivant : pour tout $n$, la valeur 1 ne peut pas être obtenue comme limite d'une suite strictement croissante de nombres contenus dans $\mathcal{T}_{n}$. Dans une autre perspective, nous interprétons la conjecture ACC comme une propriété de semi-continuité de seuils log canoniqes des séries formelles.

\section{Introduction}

Let $k$ be an algebraically closed field of characteristic zero. We consider pairs of the form $(X, Y)$, where $X$ is a smooth variety defined over $k$ and $Y \subseteq X$ is a nonempty closed subscheme. For every integer $n \geq 0$, we are interested in the set of all possible log canonical

The first author was partially supported by NSF grant DMS 0548325, and the second author was partially supported by NSF grant DMS 0500127 and a Packard Fellowship. 
thresholds in dimension $n$

$$
\mathcal{T}_{n}(k):=\{\operatorname{lct}(X, Y) \mid X \text { smooth over } k \text { of dimension } n, \varnothing \neq Y \subseteq X\} \subseteq \mathbf{R},
$$

where we make the convention that $\operatorname{lct}(X, X)=0$. It is well-known that $\mathcal{T}_{n}(k) \subseteq \mathbf{Q}$. Note that $\mathcal{T}_{0}(k)=\{0\}$ and $\mathcal{T}_{n-1}(k) \subseteq \mathcal{T}_{n}(k)$ for every $n \geq 1$.

There are two fundamental questions regarding the accumulation points (in $\mathbf{R}$ ) of these sets.

Conjecture 1.1. - For every $n$, the set $\mathcal{T}_{n}(k)$ has no points of accumulation from below.

CONJECTURE 1.2. - For every $n \geq 1$, the set of points of accumulation from above of $\mathcal{T}_{n}(k)$ is equal to $\mathcal{T}_{n-1}(k)$.

In particular, the two conjectures predict that every $\mathcal{T}_{n}(k)$ is closed, and that the set of its accumulation points is equal to $\mathcal{T}_{n-1}(k)$. In fact, both conjectures have stronger formulations, in which $\mathcal{T}_{n}(k)$ is defined under weaker conditions on the singularities of the ambient variety $X$.

Conjecture 1.1, known as the ACC Conjecture, was formulated by Shokurov in [18], where it was proved for $n=2$ (in the more general context, alluded to in the previous paragraph). Alexeev proved it for $n=3$ in [1]. The main interest in this conjecture comes from its implications to the Minimal Model Program, more precisely, to the Termination of Flips Conjecture (see [3] for a precise statement). Conjecture 1.2 above was suggested by Kollár in [11]. It was shown in [15] that Conjecture 1.2 follows if one assumes the Minimal Model Program and a conjecture of Alexeev-Borisov-Borisov on the boundedness of $\mathbf{Q}$-Fano varieties. In particular, it is known to hold (in a more general formulation) for $n \leq 3$.

It is not hard to see that the set $\mathcal{T}_{n}(k)$ is independent of $k$ (see Propositions 3.1 and 3.3 below). From now on we simply write $\mathcal{T}_{n}$ instead of $\mathcal{T}_{n}(k)$. Our main goal is to prove Conjecture 1.2, as well as the fact that $\mathcal{T}_{n}$ is closed. We state our main results in the following order.

Theorem 1.3. - For every $n$, the set $\mathcal{T}_{n}$ is closed in $\mathbf{R}$.

Since $\mathcal{T}_{n} \subseteq \mathbf{Q} \cap[0, n]$, this immediately implies the following useful property.

COROLlaRy 1.4. - Every limit of log canonical thresholds on smooth varieties of bounded dimension is a rational number.

THEOREM 1.5. - For every $n \geq 1$, the set of points of accumulation from above of $\mathcal{T}_{n}$ is equal to $\mathcal{T}_{n-1}$.

We mention that there are versions of these results when instead of arbitrary subschemes we consider only hypersurfaces. Suppose that $\mathcal{H} \mathcal{T}_{n} \subseteq \mathcal{T}_{n}$ is defined by considering only pairs $(X, Y)$, where $Y$ is locally defined by one equation. In this case $\mathcal{H} \mathcal{T}_{n}=\mathcal{T}_{n} \cap[0,1]$, hence $\mathcal{H} \mathcal{T}_{n}$ is closed, too, and the set of points of accumulation from above of $\mathcal{H} \mathcal{T}_{n}$ is equal to $\mathcal{H} \mathcal{T}_{n-1} \backslash\{1\}$.

Since $\mathcal{H} \mathcal{T}_{n} \subseteq \mathcal{T}_{n} \subseteq n \cdot \mathcal{H} \mathcal{T}_{n}$, it follows that Conjecture 1.1 holds if and only if, for every $n$, the set $\mathcal{H} \mathcal{T}_{n}$ has no points of accumulation from below. As a consequence of Corollary 1.4, we show that the conjecture can be reduced to a special case.

$4^{\text {e }}$ SÉRIE - TOME $42-2009-\mathrm{N}^{\mathrm{o}} 3$ 
COROLlary 1.6. - Conjecture 1.1 holds for every $n$ if and only if the following special case holds: for every $n$, there is $\delta_{n} \in(0,1)$ such that $\mathcal{H} \mathcal{T}_{n} \cap\left(\delta_{n}, 1\right)=\varnothing$.

In a different direction, we investigate the ACC Conjecture using the Zariski topology on the set of formal power series.

Proposition 1.7. - Conjecture 1.1 holds for $n$ if and only if, assuming that $k$ is uncountable, for every $c$ there is an integer $N(n, c)$ such that the condition for $f$ to lie in

$$
\mathcal{R}_{n}(c):=\left\{f \in k \llbracket x_{1}, \ldots, x_{n} \rrbracket \mid f(0)=0, \operatorname{lct}(f) \geq c\right\}
$$

depends only on the truncation of $f$ up to degree $N(n, c)$.

In fact, we will see that the set $\mathcal{R}_{n}(c)$ has the property in the above proposition if and only if it is open inside the maximal ideal with respect to the Zariski topology on $k \llbracket x_{1}, \ldots, x_{n} \rrbracket$ (see §5). Furthermore, Corollary 1.6 implies that in order to prove the ACC Conjecture for every $n$, it is enough to prove the assertion in the proposition only for the sets $\mathcal{R}_{n}(1)$.

The main ingredient in the proof of the above theorems is given by non-standard methods. This approach is very natural in this context, when one wants to encode a sequence of polynomials (or ideals) in a single object. In our case, we start with a sequence of ideals $\mathfrak{a}_{m} \subset k\left[x_{1}, \ldots, x_{n}\right]$ whose $\log$ canonical thresholds converge to some $c \in \mathbf{R}$. Ultrafilter constructions give non-standard extensions of our algebraic structures: we get a field ${ }^{*} k$ containing $k$ and a ring ${ }^{*}\left(k\left[x_{1}, \ldots, x_{n}\right]\right)$ containing $k\left[x_{1}, \ldots, x_{n}\right]$. Moreover, there is a truncation map from ${ }^{*}\left(k\left[x_{1}, \ldots, x_{n}\right]\right)$ to the formal power series ring ${ }^{*} k \llbracket x_{1}, \ldots, x_{n} \rrbracket$. Our sequence of ideals determines an ideal $\left[\mathfrak{a}_{m}\right] \subset{ }^{*}\left(k\left[x_{1}, \ldots, x_{n}\right]\right)$ whose image in ${ }^{*} k \llbracket x_{1}, \ldots, x_{n} \rrbracket$ we denote by $\widetilde{\mathfrak{a}}$. Our key result is that $\operatorname{lct}(\tilde{\mathfrak{a}})=c$. After possibly replacing ${ }^{*} k$ by a larger field $K$, we obtain an ideal in a polynomial ring over $K$ whose $\log$ canonical threshold is $c$. Since $\mathcal{T}_{n}(k)$ is independent of $k$, we get the conclusion of Theorem 1.3. If the sequence $\left\{c_{m}\right\}_{m}$ is strictly decreasing, then we conclude that the limit is actually a log canonical threshold in a smaller dimension via a more careful analysis of the singularities of the ideal $\tilde{\mathfrak{a}}$. We mention that non-standard methods were also employed in [5] to study the sets of $F$-pure thresholds of hypersurfaces in positive characteristic (though in that case one could only obtain the analogue of Theorem 1.3 above).

As it should be apparent from the above sketch of the proof, we need to work with log canonical thresholds of ideals in formal power series rings. The familiar framework for studying such invariants is that of schemes of finite type over a field. However, since resolutions of singularities are available for arbitrary excellent schemes (see [19]), it is not hard to extend the theory of log canonical thresholds and multiplier ideals to such a general setting. We explain this extension in detail in the next section.

In $\S 3$ we discuss some elementary properties of the sets $\mathcal{T}_{n}$, in particular the independence of the base field. The proofs of the main results are contained in $\$ 4$. In $\S 5$ we make some comments on Conjecture 1.1, proving Corollary 1.6 and Proposition 1.7. The proposition follows from a basic property of cylinders in the ring of formal power series. This interpretation of the ACC Conjecture illustrates once more that formal power series provide the natural setting when considering sequences of log canonical thresholds.

After the first version of this article was made public, János Kollár gave a new proof of the above theorems using an infinite sequence of approximations and field extensions in place 
of non-standard methods (at the core the two proofs are the same). In fact, making use of results from [4], he obtains a stronger version of Theorem 1.5, showing that all accumulation points of the set of log canonical thresholds in dimension $n$ (not just the limits of decreasing sequences) are log canonical thresholds in dimension $n-1$.

\subsection{Acknowledgments}

The second author is indebted to Caucher Birkar who introduced him to non-standard constructions. We are grateful to Jarek Włodarczyk for various discussions on resolution of singularities, and to Matthias Aschenbrenner and Pierre Milman for pointing out some key references. We would also like to acknowledge the comments and suggestions we received from an anonymous referee. Our project began at the AIM workshop on "Numerical invariants of singularities and higher-dimensional algebraic varieties". We would like to thank AIM for providing a stimulating environment.

\section{Multiplier ideals on excellent schemes}

Our goal in this section is to develop the theory of multiplier ideals and log canonical thresholds for ideals on a regular excellent scheme of characteristic zero. We will apply this theory when the ambient space is either a smooth scheme of finite type over a field or the spectrum of a formal power series ring over a field. All our schemes have characteristic zero, that is, they are schemes over $\operatorname{Spec}(\mathbf{Q})$.

Recall that a Noetherian ring $A$ is excellent if the following hold:

1) For every prime ideal $\mathfrak{p}$ in $A$, the completion morphism $A_{\mathfrak{p}} \rightarrow \widehat{A_{\mathfrak{p}}}$ has geometrically regular fibers.

2) For every $A$-algebra of finite type $B$, the regular locus of $\operatorname{Spec}(B)$ is open.

3) $A$ is universally catenary.

For the basics on excellent rings we refer to [14]. It is known that every algebra of finite type over an excellent ring is excellent, and that all complete Noetherian local rings are excellent. A Noetherian scheme $X$ is excellent if it admits an open cover by spectra of excellent rings.

The key ingredient in building the theory of multiplier ideals is the existence of log resolutions of singularities. It was shown in [19] that Hironaka's Theorem giving existence of resolutions for integral schemes of finite type over a field implies the following general statement (in fact, the result in loc. cit. holds for quasi-excellent schemes, but we do not need this generality).

Theorem 2.1 ([19]). - Let $X$ be an integral, excellent scheme of characteristic zero, and let $Y \hookrightarrow X$ be a proper closed subscheme. There is a proper, birational morphism $f: X^{\prime} \rightarrow X$ with the following properties:

i) $X^{\prime}$ is a regular scheme.

ii) The inverse image $f^{-1}(Y)$ is a divisor with simple normal crossings.

Moreover, if $U \subseteq X$ is an open subset of $X$ that is regular, and such that $U \cap Y=\varnothing$, then one can take $f$ to be an isomorphism over $U$. We note that while the statement in loc. cit. only gives that $f^{-1}(Y)$ has normal crossings, further resolving to a simple normal crossings divisor is standard.

$4^{\text {e }}$ SÉRIE - TOME $42-2009-\mathrm{N}^{\mathrm{o}} 3$ 
From now on we assume that all our schemes are excellent, of characteristic zero. We want to define the relative canonical divisor $K_{Z / X}$ for a proper birational morphism $f: Z \rightarrow X$ between two regular schemes. We consider the ideal $J$ on $Z$ defined as the Fitting ideal $\operatorname{Fitt}^{0}\left(\Omega_{Z / X}\right)$. We first show that this ideal can be computed locally in a similar way with the case of schemes of finite type over a field.

Given $z \in Z$ and $x=f(z)$, we consider the injective homomorphism of regular local rings $\varphi: \mathcal{O}_{X, x} \rightarrow \mathcal{O}_{Z, z}$. Since this morphism induces an isomorphism of rings of fractions, and since our rings are in particular universally catenary, it follows from the dimension formula (see Theorem 15.6 in [14]) that

$$
\operatorname{dim}\left(\mathcal{O}_{X, x}\right)=\operatorname{dim}\left(\mathcal{O}_{Z, z}\right)+\operatorname{trdeg}(k(z) / k(x)) .
$$

If we choose local parameters $t_{1}, \ldots, t_{r}$ for $\mathcal{O}_{X, x}$, and $u_{1}, \ldots, u_{s}$ for $\mathcal{O}_{Z, z}$, then the ring homomorphism induced by $\varphi$ at the level of completions can be described as

$$
\widehat{\varphi}: k(x) \llbracket t_{1}, \ldots, t_{r} \rrbracket \rightarrow k(z) \llbracket u_{1}, \ldots, u_{s} \rrbracket .
$$

Note that $k(z)$ is a separable extension of $k(x)$, with $\operatorname{trdeg}(k(z) / k(x))=r-s$. A basis of $\operatorname{Der}_{k(x)}(k(z))$ induces derivations $D_{1}, \ldots, D_{r-s}$ of $k(z) \llbracket u_{1}, \ldots, u_{s} \rrbracket$ over $k(x)$, by acting on coefficients. If we put $\varphi_{i}:=\widehat{\varphi}\left(t_{i}\right)$ and $D_{r-s+j}=\partial / \partial u_{j}$ for $1 \leq j \leq s$, then we see that $J \cdot \widehat{\mathcal{O}_{Z, z}}$ is generated by $\operatorname{det}\left(D_{i}\left(\varphi_{j}\right)\right)_{1 \leq i, j \leq r}$. In particular, $J$ is a locally principal ideal around $z \in Z$. We denote by $K_{Z / X}$ the effective divisor corresponding to $J$. It defines the locus where the morphism $f$ is not étale. Its complement is the inverse image of an open subset $U$ of $X$, such that $f^{-1}(U) \rightarrow U$ is an isomorphism.

Suppose now that $X$ is a regular connected scheme. If $Y$ is a closed, proper subscheme of $X$, a $\log$ resolution $f: X^{\prime} \rightarrow X$ of $(X, Y)$ is a morphism as in Theorem 2.1, such that in addition the union of $f^{-1}(Y)$ with the exceptional locus of $f$ has simple normal crossings. (Note that this exceptional locus is automatically a divisor, being equal to the support of $K_{X^{\prime} / X}$.)

We see that every $f: X^{\prime} \rightarrow X$ as in Theorem 2.1 is dominated by some $g: X^{\prime \prime} \rightarrow X^{\prime}$ such that $f \circ g$ is a $\log$ resolution. Indeed, it is enough to apply the theorem for $\left(X^{\prime}, D\right)$, where $D=f^{-1}(Z)+K_{X^{\prime} / X}$ to get $g$ that is an isomorphism over $X^{\prime} \backslash \operatorname{Supp}(D)$ (in this case, the exceptional locus of $f \circ g$ is contained in $g^{-1}(D)$ ).

Furthermore, the above assertion implies that every two log resolutions of $(X, Y)$ are dominated by a third one.

Let $X$ be a regular, connected excellent scheme of characteristic zero. Suppose that $Y$ is a proper closed subscheme of $X$, and let $\mathfrak{a}$ be the ideal sheaf defining $Y$. If $\lambda \in \mathbf{R}_{\geq 0}$, then the multiplier ideal sheaf $\mathcal{J}\left(\mathfrak{a}^{\lambda}\right)$ is defined as follows.

Consider a $\log$ resolution $f: X^{\prime} \rightarrow X$ of the pair $(X, Y)$. If we denote $f^{-1}(\mathfrak{a})=$ $\mathcal{O}_{X^{\prime}}(-F)$, then

$$
\mathcal{J}\left(\mathfrak{a}^{\lambda}\right):=f_{*} \mathcal{O}_{X^{\prime}}\left(K_{X^{\prime} / X}-\lfloor\lambda F\rfloor\right) .
$$

Since $K_{X^{\prime} / X}$ is an effective exceptional divisor, we have $f_{*} \mathcal{O}_{X^{\prime}}\left(K_{X^{\prime} / X}\right)=\mathcal{O}_{X}$, and therefore $\mathcal{J}\left(\mathfrak{a}^{\lambda}\right)$ is a (coherent) sheaf of ideals in $\mathcal{O}_{X}$.

This is, of course, consistent with the usual definition of multiplier ideals when $X$ is of finite type over a field. Our reference for the basic results on multiplier ideals is [13]. Most of 
the results therein extend almost verbatim to our setting. We state the results for completeness, but we give detailed proofs only when there is a significant difference from the familiar case.

Proposition 2.2. - The definition of $\mathcal{J}\left(\mathfrak{a}^{\lambda}\right)$ does not depend on the choice of a log resolution.

Proof. - The argument follows as in [13], Thm. 9.2.18, using the fact that every two log resolutions can be dominated by a third one, and the following consequence of our local computation of $K_{X^{\prime} / X}$. If $E$ is a smooth prime divisor on $X^{\prime}$, and $D_{1}, \ldots, D_{r}$ are simple normal crossings divisors such that $E$ appears with coefficient $a_{i} \geq 1$ in $f^{*}\left(D_{i}\right)$, then $E$ appears with coefficient $\geq a_{1}+\cdots+a_{r}-1$ in $K_{X^{\prime} / X}$.

Note that if $\mathfrak{a}=\mathcal{O}_{X}$, then $\mathcal{J}\left(\mathfrak{a}^{\lambda}\right)=\mathcal{O}_{X}$ for every $\lambda \geq 0$. We make the convention that if $\mathfrak{a}=0$, then $\mathcal{J}\left(\mathfrak{a}^{\lambda}\right)=0$ for every $\lambda \geq 0$. The following easy properties follow immediately from the definition.

Proposition 2.3. - Let $X$ be a scheme as above, and let $\mathfrak{a}$ be a (coherent) sheaf of ideals on $X$.

1) If $\lambda<\mu$, then $\mathcal{J}\left(\mathfrak{a}^{\mu}\right) \subseteq \mathcal{J}\left(\mathfrak{a}^{\lambda}\right)$.

2) For every $\lambda \in \mathbf{R}_{\geq 0}$, there is $\varepsilon>0$ such that $\mathcal{J}\left(\mathfrak{a}^{\mu}\right)=\mathcal{J}\left(\mathfrak{a}^{\lambda}\right)$ for every $\mu \in[\lambda, \lambda+\varepsilon)$.

3) If $\mathfrak{b} \subseteq \mathfrak{a}$, then $\mathcal{J}\left(\mathfrak{b}^{\lambda}\right) \subseteq \mathcal{J}\left(\mathfrak{a}^{\lambda}\right)$.

4) If $\mathfrak{a} \neq 0$, then $\mathcal{J}\left(\mathfrak{a}^{0}\right)=\mathcal{O}_{X}$.

Suppose now that $\mathfrak{a} \neq 0$ and $\mathfrak{a} \neq \mathcal{O}_{X}$. A positive number $\lambda$ is called a jumping number of $\mathfrak{a}$ if $\mathcal{J}\left(\mathfrak{a}^{\lambda}\right) \neq \mathcal{J}\left(\mathfrak{a}^{\lambda^{\prime}}\right)$ for every $\lambda^{\prime}<\lambda$. It is clear that $\mathfrak{a}$ has jumping numbers.

Consider a $\log$ resolution $f: X^{\prime} \rightarrow X$, and the divisors $F$ and $K_{X^{\prime} / X}$, where $f^{-1}(\mathfrak{a})=$ $\mathcal{O}(-F)$. Write

$$
F=\sum_{i=1}^{r} a_{i} E_{i}, K_{X^{\prime} / X}=\sum_{i=1}^{r} \kappa_{i} E_{i} .
$$

It is clear that if $\lambda$ is a jumping number of $\mathfrak{a}$, then there is $i$ such that $a_{i} \lambda$ is an integer. In particular, all jumping numbers are rational numbers. Moreover, the jumping numbers of a given ideal $\mathfrak{a}$ form a discrete subset of $\mathbf{R}_{+}$.

The smallest jumping number of $\mathfrak{a}$ is called the log canonical threshold of $\mathfrak{a}$, and it is denoted by lct $(\mathfrak{a})$. It is the smallest $\lambda$ such that $\mathcal{J}\left(\mathfrak{a}^{\lambda}\right) \neq \mathcal{O}_{X}$. With the notation in (1), we have

$$
\operatorname{lct}(\mathfrak{a})=\min _{i} \frac{\kappa_{i}+1}{a_{i}} .
$$

It follows from Proposition 2.3 3) that if $\mathfrak{b} \subseteq \mathfrak{a}$, then $\operatorname{lct}(\mathfrak{b}) \leq \operatorname{lct}(\mathfrak{a})$. It is natural to make the convention that $\operatorname{lct}(0)=0$ and $\operatorname{lct}\left(\mathcal{O}_{X}\right)=\infty$.

Proposition 2.4. - If $\overline{\mathfrak{a}}$ denotes the integral closure of the ideal $\mathfrak{a}$, then $\mathcal{J}\left(\mathfrak{a}^{\lambda}\right)=\mathcal{J}\left(\overline{\mathfrak{a}}^{\lambda}\right)$ for every $\lambda$. In particular, we have $\operatorname{lct}(\mathfrak{a})=\operatorname{lct}(\overline{\mathfrak{a}})$.

Proof. - This is an immediate consequence of the fact that if $f: X^{\prime} \rightarrow X$ is a log resolution of both $\mathfrak{a}$ and $\overline{\mathfrak{a}}$, then $f^{-1}(\mathfrak{a})=f^{-1}(\overline{\mathfrak{a}})$. See also [13], §9.6.A. 
It is convenient to define also a local version of the $\log$ canonical threshold: if $\xi \in X$ is a (not necessarily closed) point in $V(\mathfrak{a})$, then we define

$$
\operatorname{lct}_{\xi}(\mathfrak{a}):=\min \left\{\operatorname{lct}\left(\left.\mathfrak{a}\right|_{U}\right) \mid U \subseteq X \text { open, } \xi \in U\right\} .
$$

Using the notation in (1), we have

$$
\operatorname{lct}_{\xi}(\mathfrak{a})=\min \left\{\left(\kappa_{i}+1\right) / a_{i} \mid \xi \in f\left(E_{i}\right)\right\} .
$$

Note that if $X=\operatorname{Spec}(R)$, where $R$ is a local ring, then $X$ has only one closed point $x_{0}$, and $\operatorname{lct}_{x_{0}}(\mathfrak{a})=\operatorname{lct}(\mathfrak{a})$.

The log canonical threshold locus $\operatorname{LCT}(\mathfrak{a})$ of $\mathfrak{a}$ is the closed subset of $X$ where $\mathcal{J}\left(\mathfrak{a}^{\operatorname{lct}(\mathfrak{a})}\right)$ vanishes. More generally, for every (not necessarily closed) point $\xi$ on $X$, we denote by $\operatorname{LCT}_{\xi}(\mathfrak{a})$ the support of the closed subscheme of $X$ defined by $\mathcal{J}\left(\mathfrak{a}^{\operatorname{lct}_{\xi}(\mathfrak{a})}\right)$. With the notation in (1), we have

$$
\operatorname{LCT}(\mathfrak{a})=\bigcup_{\frac{\kappa_{i}+1}{a_{i}}=\operatorname{lct}(\mathfrak{a})} f\left(E_{i}\right) \text { and } \operatorname{LCT}_{\xi}(\mathfrak{a})=\bigcup_{\frac{\kappa_{i}+1}{a_{i}} \leq \operatorname{lct}_{\xi}(\mathfrak{a})} f\left(E_{i}\right) .
$$

Note that $\operatorname{LCT}_{\xi}(\mathfrak{a}) \cap U=\operatorname{LCT}\left(\left.\mathfrak{a}\right|_{U}\right)$ for a suitable open neighborhood $U$ of $\xi$.

Suppose that $X$ is a scheme as above, and let $\mathfrak{a}$ be a nonzero ideal sheaf on $X$. We consider a point $\xi \in X$, and let $\mathfrak{p}$ denote the ideal defining the closed set $\overline{\{\xi\}}$ (with the reduced scheme structure). Suppose that $\xi$ lies in the support of the subscheme defined by $\mathfrak{a}$ (that is, $\mathfrak{p} \supseteq \mathfrak{a}$ ). Since $\mathfrak{a} \subseteq \mathfrak{a}+\mathfrak{p}^{d}$, we clearly have

$$
\operatorname{lct}_{\xi}\left(\mathfrak{a}+\mathfrak{p}^{d}\right) \geq \operatorname{lct}_{\xi}(\mathfrak{a}) \quad \text { for all } d \geq 1 .
$$

In fact, the following key property holds.

Proposition 2.5. - With the above notation, we have

$$
\lim _{d \rightarrow \infty} \operatorname{lct}_{\xi}\left(\mathfrak{a}+\mathfrak{p}^{d}\right)=\operatorname{lct}_{\xi}(\mathfrak{a}) .
$$

Moreover, if $\overline{\{\xi\}}$ is an irreducible component of $\operatorname{LCT}_{\xi}(\mathfrak{a})$, then $\operatorname{lct}_{\xi}\left(\mathfrak{a}+\mathfrak{p}^{d}\right)=\operatorname{lct}_{\xi}(\mathfrak{a})$ for all $d \gg 0$.

We first prove the following lemma. Recall that a divisor over $X$ is a prime divisor $E$ on some $X^{\prime}$, where we have a proper birational morphism $f: X^{\prime} \rightarrow X$, with $X^{\prime}$ regular. The center of $E$ on $X$ is $c_{X}(E):=f(E)$. Such a divisor defines a valuation $\operatorname{ord}_{E}$ of the function field of $X$. In particular, we may define $\operatorname{ord}_{E}\left(K_{X^{\prime} / X}\right)$ and $\operatorname{ord}_{E}(\mathfrak{a})$ in the natural way. Note that if we use the notation in (1), then $\operatorname{ord}_{E_{i}}\left(K_{X^{\prime} / X}\right)=\kappa_{i}$ and $\operatorname{ord}_{E_{i}}(\mathfrak{a})=a_{i}$.

Lemma 2.6. - With the notation in Proposition 2.5, we have

$$
\operatorname{lct}_{\xi}(\mathfrak{a})=\inf _{c_{X}(E)=\{\xi\}} \frac{\operatorname{ord}_{E}\left(K_{X^{\prime} / X}\right)+1}{\operatorname{ord}_{E}(\mathfrak{a})},
$$

where the infimum is taken over all divisors $E$ over $X$ whose center on $X$ is equal to $\overline{\{\xi\}}$. Moreover, if $\overline{\{\xi\}}$ is an irreducible component of $\operatorname{LCT}_{\xi}(\mathfrak{a})$, then there is a divisor $E$ with center $\overline{\{\xi\}}$ on $X$, such that

$$
\operatorname{lct}_{\xi}(\mathfrak{a})=\frac{\operatorname{ord}_{E}\left(K_{X^{\prime} / X}\right)+1}{\operatorname{ord}_{E}(\mathfrak{a})} .
$$


Proof. - Note first that the infimum in (3) is $\geq \operatorname{lct}_{\xi}(\mathfrak{a})$ by definition. In order to prove the other inequality, the argument follows as in [12, (17.1.1.3)]. Let us consider a log resolution $f: X^{\prime} \rightarrow X$ for $\mathfrak{a} \cdot \mathfrak{p}$ (recall that $\mathfrak{p}$ is the ideal defining $\overline{\{\xi\}}$ ). With the notation in (1), fix a prime divisor $F=E_{i}$ on $X^{\prime}$ such that $\xi \in c_{X}(F)$ and $\operatorname{lct}_{\xi}(\mathfrak{a})=\frac{\kappa_{i}+1}{a_{i}}$.

If $c_{X}(F)=\overline{\{\xi\}}$, then we are done. Otherwise, by construction, $f^{-1}(\overline{\{\xi\}})$ is a union of divisors having simple normal crossings with $F$. Hence we may choose such a divisor $F_{0}$, with $F \cap F_{0}$ containing a point $\xi^{\prime} \in f^{-1}(\xi)$. This implies that $f\left(F \cap F_{0}\right)=\overline{\{\xi\}}$. We define recursively $F_{m}$ for $m \geq 1$, as follows. Let $X_{1}^{\prime}$ be the blow-up of $X^{\prime}$ along $F \cap F_{0}$, and $F_{1}$ be a component of the exceptional divisor whose image in $X^{\prime}$ contains $\xi^{\prime}$. In general, we denote by $X_{m}^{\prime}$ the blow-up of $X_{m-1}^{\prime}$ along the intersection of $F_{m-1}$ with the proper transform of $F$, and by $F_{m}$ the component of the exceptional divisor whose image in $X^{\prime}$ contains $\xi^{\prime}$. It is clear that $c_{X}\left(F_{m}\right)=\overline{\{\xi\}}$ for every $m$. On the other hand, a standard computation gives

$$
\begin{gathered}
\operatorname{ord}_{F_{m}}(\mathfrak{a})=m \cdot \operatorname{ord}_{F}(\mathfrak{a})+\operatorname{ord}_{F_{0}}(\mathfrak{a}), \\
1+\operatorname{ord}_{F_{m}}\left(K_{X_{m}^{\prime} / X}\right)=m\left(1+\operatorname{ord}_{F}\left(K_{X^{\prime} / X}\right)\right)+\left(1+\operatorname{ord}_{F_{0}}\left(K_{X^{\prime} / X}\right)\right) .
\end{gathered}
$$

Therefore we get (3), since

$$
\lim _{m \rightarrow \infty} \frac{1+\operatorname{ord}_{F_{m}}\left(K_{X_{m}^{\prime} / X}\right)}{\operatorname{ord}_{m}(\mathfrak{a})}=\operatorname{lct}_{\xi}(\mathfrak{a}) .
$$

The second assertion, when $\overline{\{\xi\}}$ is an irreducible component of $\operatorname{LCT}_{\xi}(\mathfrak{a})$, follows from (2). Indeed, note that if $\xi \in f\left(E_{i}\right)$, then by definition we have

$$
\operatorname{lct}_{\xi}(\mathfrak{a}) \leq \frac{\operatorname{ord}_{E_{i}}\left(K_{X^{\prime} / X}\right)+1}{\operatorname{ord}_{E_{i}}(\mathfrak{a})}
$$

and the inequality is strict if $\overline{\{\xi\}}$ is strictly contained in $f\left(E_{i}\right)$.

Therefore every irreducible component of $\operatorname{LCT}_{\xi}(\mathfrak{a})$ containing $\xi$ is of the form $f\left(E_{i}\right)$ for some $E_{i}$ that achieves equality in (4).

Proof of Proposition 2.5. - By the lemma, we see that for every $\varepsilon>0$ there is a divisor $E$ on some $X^{\prime}$ over $X$ having center $\overline{\{\xi\}}$ and such that

$$
\frac{\operatorname{ord}_{E}\left(K_{X^{\prime} / X}\right)+1}{\operatorname{ord}_{E}(\mathfrak{a})} \leq \operatorname{lct}_{\xi}(\mathfrak{a})+\varepsilon
$$

Since $\mathfrak{p}$ is the ideal defining $\overline{\{\xi\}}$, we have $\operatorname{ord}_{E}(\mathfrak{p}) \geq 1$. It follows that if $d>\operatorname{ord}_{E}(\mathfrak{a})$, then $\operatorname{ord}_{E}\left(\mathfrak{a}+\mathfrak{p}^{d}\right)=\operatorname{ord}_{E}(\mathfrak{a})$. Hence for all such $d$ we have

$$
\operatorname{lct}_{\xi}\left(\mathfrak{a}+\mathfrak{p}^{d}\right) \leq \frac{\operatorname{ord}_{E}\left(K_{X^{\prime} / X}\right)+1}{\operatorname{ord}_{E}\left(\mathfrak{a}+\mathfrak{p}^{d}\right)}=\frac{\operatorname{ord}_{E}\left(K_{X^{\prime} / X}\right)+1}{\operatorname{ord}_{E}(\mathfrak{a})} \leq \operatorname{lct}_{\xi}(\mathfrak{a})+\varepsilon .
$$

Therefore $\lim \sup _{d \rightarrow \infty} \operatorname{lct}_{\xi}\left(\mathfrak{a}+\mathfrak{p}^{d}\right) \leq \operatorname{lct}_{\xi}(\mathfrak{a})$. Since, as we have already observed, $\operatorname{lct}_{\xi}\left(\mathfrak{a}+\mathfrak{p}^{d}\right) \geq \operatorname{lct}_{\xi}(\mathfrak{a})$ for every $d \geq 1$, we conclude that the limit exists and is equal to $\operatorname{lct}_{\xi}(\mathfrak{a})$.

Regarding the second assertion, if $\overline{\{\xi\}}$ is an irreducible component of $\operatorname{LCT}_{\xi}(\mathfrak{a})$, then by the second part of the lemma we can find an $E$ with center $\overline{\{\xi\}}$ such that

$$
\frac{\operatorname{ord}_{E}\left(K_{X^{\prime} / X}\right)+1}{\operatorname{ord}_{E}(\mathfrak{a})}=\operatorname{lct}_{\xi}(\mathfrak{a}),
$$

and thus we obtain that $\operatorname{lct}_{\xi}\left(\mathfrak{a}+\mathfrak{p}^{d}\right)=\operatorname{lct}_{\xi}(\mathfrak{a})$ for all $d>\operatorname{ord}_{E}(\mathfrak{a})$. 
Our next result allows us to reduce the computation of multiplier ideals and log canonical thresholds to the case when $X$ is the spectrum of a complete regular local ring of characteristic zero.

Proposition 2.7. - Let $X$ be a regular, connected excellent scheme of characteristic zero. Suppose that $\xi \in X$ is a (not necessarily closed) point, and let $\widehat{\mathcal{O}_{X, \xi}}$ be the completion of the local ring at $\xi$. If $h: \operatorname{Spec}\left(\widehat{\mathcal{O}_{X, \xi}}\right) \rightarrow X$ is the canonical morphism, then for every nonzero sheaf of ideals $\mathfrak{a}$ on $X$ such that $\xi$ lies in the support of the subscheme defined by $\mathfrak{a}$, and for every $\lambda \in \mathbf{R}_{+}$we have

$$
\mathcal{J}\left(\mathfrak{a}^{\lambda}\right) \cdot \widehat{\mathcal{O}_{X, \xi}}=\mathcal{J}\left(\left(\mathfrak{a} \cdot \widehat{\mathcal{O}_{X, \xi}}\right)^{\lambda}\right) .
$$

In particular, we have $\operatorname{lct}_{\xi}(\mathfrak{a})=\operatorname{lct}\left(\mathfrak{a} \cdot \widehat{\mathcal{O}_{X, \xi}}\right)$.

We will make use, in particular, of the following special case.

COROLlary 2.8. - Let $X$ and $\mathfrak{a}$ be as in the theorem. If $\xi$ is the generic point of an irreducible component of $\operatorname{LCT}(\mathfrak{a})$, then $\operatorname{lct}(\mathfrak{a})=\operatorname{lct}\left(\mathfrak{a} \cdot \widehat{\mathcal{O}_{X, \xi}}\right)$, and $\operatorname{LCT}\left(\mathfrak{a} \cdot \widehat{\mathcal{O}_{X, \xi}}\right)$ consists only of the closed point.

Proof of Proposition 2.7. - Let $W=\operatorname{Spec}\left(\widehat{\mathcal{O}_{X, \xi}}\right)$, and denote by $Y$ and $Z$ the subschemes of $X$ and $W$, respectively defined by $\mathfrak{a}$ and $\mathfrak{a} \cdot \widehat{\mathcal{O}_{X, \xi}}$. Let $f: X^{\prime} \rightarrow X$ be a $\log$ resolution of $(X, Y)$. If we consider the Cartesian diagram

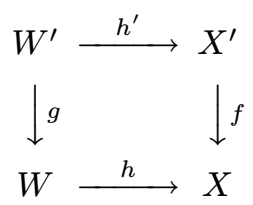

then $g$ is a $\log$ resolution of $(W, Z)$. Indeed, it is clear that $g$ is proper, and if $f$ is an isomorphism over the nonempty open subset $U$, then $g$ is an isomorphism over $h^{-1}(U)$, which is nonempty. Furthermore, $W^{\prime}$ is a regular scheme. In order to see this, it is enough to show that all fibers of $h^{\prime}$ are regular (note that $h$ is flat, and therefore $h^{\prime}$ is flat, too, and we may apply Thm. 23.7 in [14]). This in turn follows from the fact that the morphism $\mathcal{O}_{X, \xi} \rightarrow \overline{\mathcal{O}_{X, \xi}}$ has geometrically regular fibers (see the first property in the definition of excellent rings).

Using the same argument, we see that if $x_{1}, \ldots, x_{n}$ are algebraic coordinates in an open subset $V$ of $X^{\prime}$, then their pull-backs to $h^{\prime-1}(V)$ give an algebraic system of coordinates in this open subset of $W^{\prime}$. Note also that $K_{W^{\prime} / W}=h^{\prime *}\left(K_{X / X}\right)$. Putting these together, we see that $K_{W^{\prime} / W}+g^{-1}(Z)$ is a divisor with simple normal crossings on $W^{\prime}$, and therefore $g$ is a $\log$ resolution of $(W, Z)$. Moreover, if $F=f^{-1}(Y)$, then $h^{\prime *}(F)=g^{-1}(Z)$, and flat base change gives

$\mathcal{J}\left(\left(\mathfrak{a} \cdot \widehat{\mathcal{O}_{X, \xi}}\right)^{\lambda}\right)=g_{*} \mathcal{O}_{W^{\prime}}\left(h^{\prime *}\left(K_{X^{\prime} / X}-\lfloor\lambda F\rfloor\right)\right)=h^{*} f_{*} \mathcal{O}_{X^{\prime}}\left(K_{X^{\prime} / X}-\lfloor\lambda F\rfloor\right)=\mathcal{J}\left(\mathfrak{a}^{\lambda}\right) \cdot \widehat{\mathcal{O}_{X, \xi}}$.

The assertion about log canonical thresholds is an immediate consequence, since $\operatorname{lct}_{\xi}(\mathfrak{a})=\sup \left\{\lambda \mid \mathcal{J}\left(\mathfrak{a}^{\lambda}\right) \cdot \mathcal{O}_{X, \xi}=\mathcal{O}_{X, \xi}\right\}=\sup \left\{\lambda \mid \mathcal{J}\left(\mathfrak{a}^{\lambda}\right) \cdot \widehat{\mathcal{O}_{X, \xi}}=\widehat{\mathcal{O}_{X, \xi}}\right\}=\operatorname{lct}\left(\mathfrak{a} \cdot \widehat{\mathcal{O}_{X, \xi}}\right)$. 
Proposition 2.9. - Let $K / k$ be a field extension. If $\mathfrak{a}$ is a nonzero ideal in $k\left[x_{1}, \ldots, x_{n}\right]$ whose cosupport contains the point 0 corresponding to $\left(x_{1}, \ldots, x_{n}\right)$, then

$$
\operatorname{lct}_{0}(\mathfrak{a})=\operatorname{lct}_{0}\left(\mathfrak{a} \cdot K\left[x_{1}, \ldots, x_{n}\right]\right) .
$$

Similarly, if $\mathfrak{b}$ is a nonzero proper ideal in $k \llbracket x_{1}, \ldots, x_{n} \rrbracket$, then

$$
\operatorname{lct}(\mathfrak{b})=\operatorname{lct}\left(\mathfrak{b} \cdot K \llbracket x_{1}, \ldots, x_{n} \rrbracket\right) .
$$

Proof. - Let $X=\mathbf{A}_{k}^{n}$ and $W=\mathbf{A}_{K}^{n}$, and let $h: W \rightarrow X$ denote the induced flat map. It follows as in the proof of Proposition 2.7 that if $f$ is a $\log$ resolution of $(X, \mathfrak{a})$, then we get a Cartesian diagram (5) such that $g$ is a $\log$ resolution of $\left(W, \mathfrak{a} \cdot \mathcal{O}_{W}\right)$ (the key fact is that since we are in characteristic zero, the field extension $K / k$ is separable, hence geometrically regular). As a consequence, we deduce that

$$
\mathcal{J}\left(\left(\mathfrak{a} \cdot \mathcal{O}_{W}\right)^{\lambda}\right)=\mathcal{J}\left(\mathfrak{a}^{\lambda}\right) \cdot \mathcal{O}_{W}
$$

for every $\lambda \in \mathbf{R}_{+}$, and since the homomorphism $\mathcal{O}_{X, 0} \rightarrow \mathcal{O}_{W, 0}$ is faithfully flat, we get (6).

In the formal power series case, denote by $\mathfrak{m}$ and $\mathfrak{m}^{\prime}$ the maximal ideals in $k \llbracket x_{1}, \ldots, x_{n} \rrbracket$ and $K \llbracket x_{1}, \ldots, x_{n} \rrbracket$, respectively. For every $d$, the ideal $\mathfrak{b}+\mathfrak{m}^{d}$ is generated by polynomials of degree $\leq d$, hence it is equal to $\mathfrak{b}_{d} \cdot k \llbracket x_{1}, \ldots, x_{n} \rrbracket$, where $\mathfrak{b}_{d}=\left(\mathfrak{b}+\mathfrak{m}^{d}\right) \cap k\left[x_{1}, \ldots, x_{n}\right]$. If we put $\mathfrak{b}_{d}^{\prime}=\mathfrak{b}_{d} \cdot K\left[x_{1}, \ldots, x_{n}\right]$, then it follows from (6) that $\operatorname{lct}_{0}\left(\mathfrak{b}_{d}\right)=\operatorname{lct}_{0}\left(\mathfrak{b}_{d}^{\prime}\right)$ for every $d$. On the other hand, Proposition 2.7 gives

$$
\operatorname{lct}\left(\mathfrak{b}_{d} \cdot k \llbracket x_{1}, \ldots, x_{n} \rrbracket\right)=\operatorname{lct}_{0}\left(\mathfrak{b}_{d}\right) \quad \text { and } \quad \operatorname{lct}\left(\mathfrak{b}_{d}^{\prime} \cdot K \llbracket x_{1}, \ldots, x_{n} \rrbracket=\operatorname{lct}_{0}\left(\mathfrak{b}_{d}^{\prime}\right) .\right.
$$

Using Proposition 2.5, we deduce that

$$
\operatorname{lct}(\mathfrak{b})=\lim _{d \rightarrow \infty} \operatorname{lct}_{0}\left(\mathfrak{b}_{d}\right) \quad \text { and } \operatorname{lct}\left(\mathfrak{b} \cdot K \llbracket x_{1}, \ldots, x_{n} \rrbracket\right)=\lim _{d \rightarrow \infty} \operatorname{lct}_{0}\left(\mathfrak{b}_{d}^{\prime}\right),
$$

and since $\operatorname{lct}_{0}\left(\mathfrak{b}_{d}\right)=\operatorname{lct}_{0}\left(\mathfrak{b}_{d}^{\prime}\right)$, we get (7).

We note that using Propositions 2.5 and 2.7 one can extend various results about $\log$ canonical thresholds from the familiar case of varieties over an algebraically closed field to our more general setting. We state one such result which gives a sharpening of Proposition 2.5.

Corollary 2.10. - Let $X$ be a connected, regular excellent scheme, and let $\mathfrak{a}$ be a nonzero ideal sheaf on $X$. We consider a point $\xi$ in the cosupport of $\mathfrak{a}$, and let $\mathfrak{p}$ be the ideal sheaf defining $\overline{\{\xi\}}$. If $e=\operatorname{dim}\left(\mathcal{O}_{X, \xi}\right)$, and if $\mathfrak{b}$ is an ideal sheaf on $X$ such that $\mathfrak{b} \subseteq \mathfrak{a}+\mathfrak{p}^{(d)}$ (where $\mathfrak{p}^{(d)}$ denotes the $d^{\text {th }}$ symbolic power of $\left.\mathfrak{p}\right)$, then

$$
\operatorname{lct}_{\xi}(\mathfrak{b})-\operatorname{lct}_{\xi}(\mathfrak{a}) \leq \frac{e}{d}
$$

Proof. - After replacing $\mathfrak{a}$ and $\mathfrak{b}$ by their images in $\widehat{\mathcal{O}_{X, \xi}}$, and applying Proposition 2.7, we see that we may assume that $X=\operatorname{Spec}\left(k \llbracket x_{1}, \ldots, x_{e} \rrbracket\right)$ and that $\xi=\mathfrak{m}$ is the closed point. Note that in this case the hypothesis simply says that $\mathfrak{b} \subseteq \mathfrak{a}+\mathfrak{m}^{d}$, and it is enough to show that

$$
\operatorname{lct}\left(\mathfrak{a}+\mathfrak{m}^{d}\right)-\operatorname{lct}(\mathfrak{a}) \leq \frac{e}{d} .
$$

Furthermore, using Proposition 2.9, we may replace $k$ by an algebraic closure, and therefore assume that $k$ is algebraically closed. 
If we put $\mathfrak{a}_{q}=\mathfrak{a}+\mathfrak{m}^{q}$, then by Proposition 2.5 we have

$$
\operatorname{lct}(\mathfrak{a})=\lim _{q \rightarrow \infty} \operatorname{lct}\left(\mathfrak{a}_{q}\right) \quad \text { and } \quad \operatorname{lct}\left(\mathfrak{a}+\mathfrak{m}^{d}\right)=\lim _{q \rightarrow \infty} \operatorname{lct}\left(\mathfrak{a}_{q}+\mathfrak{m}^{d}\right) .
$$

Therefore it is enough to prove the corollary when we replace $\mathfrak{a}$ by $\mathfrak{a}_{q}$. Since each $\mathfrak{a}_{q}$ is the extension of an ideal in $k\left[x_{1}, \ldots, x_{e}\right]$, it follows that it is enough to prove the corollary when $X=\mathbf{A}_{k}^{e}$ and $\xi$ is the origin 0. In this case, the assertion is well-known: it follows, for example, from the fact that

$$
\operatorname{lct}_{0}\left(\mathfrak{a}+\mathfrak{m}^{d}\right) \leq \operatorname{lct}_{0}(\mathfrak{a})+\operatorname{lct}_{0}\left(\mathfrak{m}^{d}\right)=\operatorname{lct}_{0}(\mathfrak{a})+\frac{e}{d}
$$

(see Proposition 4.7 in [16]).

Note that every complete regular local ring of characteristic zero (or more generally, containing a field) is isomorphic to a formal power series ring $k \llbracket x_{1}, \ldots, x_{n} \rrbracket$. It follows from Corollary 2.8 that the log canonical threshold of every ideal $\mathfrak{a}$ in our general setting is equal to the log canonical threshold of an ideal in a formal power series ring whose log canonical threshold locus is supported at the closed point. We now show that for every such ideal, its $\log$ canonical threshold is equal to the log canonical threshold of a zero-dimensional ideal in a polynomial ring over an algebraically closed field.

Proposition 2.11. - Let X be a connected, regular excellent scheme of dimension $N$. For every proper nonzero ideal sheaf $\mathfrak{a}$ on $X$, there is an algebraically closed field $k$ of characteristic zero and an ideal $\mathfrak{b}$ in a polynomial ring $k\left[x_{1}, \ldots, x_{n}\right]$ such that $\mathfrak{b}$ is cosupported at the origin 0 , and $\operatorname{lct}(\mathfrak{a})=\operatorname{lct}_{0}(\mathfrak{b})$. Moreover,

i) We may take $n \leq N$.

ii) If $\operatorname{dim} \operatorname{LCT}(\mathfrak{a})>0$, then we may take $n<N$.

iii) If $\operatorname{dim} \operatorname{LCT}(\mathfrak{a})=0$ and $X$ is a scheme of finite type over an algebraically closed field $K$, then we may take $k=K$.

Proof. - Let $\xi$ be the generic point of an irreducible component of $\operatorname{LCT}(\mathfrak{a})$. We fix an isomorphism $\widehat{\mathcal{O}_{X, \xi}} \cong k_{0} \llbracket x_{1}, \ldots, x_{n} \rrbracket$, where $k_{0}$ is the residue field of $\widehat{\mathcal{O}_{X, \xi}}$. By Corollary 2.8, the image of $\mathfrak{a} \cdot \widehat{\mathcal{O}_{X, \xi}}$ via this isomorphism is an ideal $\widetilde{\mathfrak{a}}$ of $k_{0} \llbracket x_{1}, \ldots, x_{n} \rrbracket \operatorname{with} \operatorname{lct}(\widetilde{\mathfrak{a}})=\operatorname{lct}(\mathfrak{a})$ and $\operatorname{LCT}(\widetilde{\mathfrak{a}})=\{\widetilde{\mathfrak{m}}\}$ (here $\widetilde{\mathfrak{m}}$ denotes the maximal ideal of $k_{0} \llbracket x_{1}, \ldots, x_{n} \rrbracket$ ). Proposition 2.5 gives $\operatorname{lct}\left(\widetilde{\mathfrak{a}}+\widetilde{\mathfrak{m}}^{d}\right)=\operatorname{lct}(\widetilde{\mathfrak{a}})$ for all $d \gg 0$, and clearly $\operatorname{LCT}\left(\widetilde{\mathfrak{a}}+\widetilde{\mathfrak{m}}^{d}\right)=\{\widetilde{\mathfrak{m}}\}$ for such $d$. We fix a sufficiently large $d$, and observe that the ideal $\widetilde{\mathfrak{a}}+\widetilde{\mathfrak{m}}^{d}$ is generated by polynomials of degree $\leq d$, hence $\widetilde{\mathfrak{a}}+\widetilde{\mathfrak{m}}^{d}=\mathfrak{c} \cdot k_{0} \llbracket x_{1}, \ldots, x_{n} \rrbracket$ for some ideal $\mathfrak{c} \subseteq k_{0}\left[x_{1}, \ldots, x_{n}\right]$. We put $\mathfrak{b}=\mathfrak{c} \cdot k\left[x_{1}, \ldots, x_{n}\right]$, where $k$ is an algebraic closure of $k_{0}$. Since $\operatorname{lct}\left(\widetilde{\mathfrak{a}}+\widetilde{\mathfrak{m}}^{d}\right)=\operatorname{lct}_{0}(\mathfrak{c})$ by Proposition 2.7, and $\operatorname{lct}_{0}(\mathfrak{b})=\operatorname{lct}_{0}(\mathfrak{c})$ by Proposition 2.9, we deduce that $\operatorname{lct}(\mathfrak{a})=\operatorname{lct}_{0}(\mathfrak{b})$.

It is clear from the above construction that $n \leq N$, and the inequality is strict if $\xi$ is not a closed point. This gives i) and ii) in the proposition. Moreover, if $X$ is of finite type over the algebraically closed field $K$, and $\xi$ is a closed point, then $k_{0}=K$, which gives iii). 


\section{Sets of log canonical thresholds}

Let $k$ be an algebraically closed field of characteristic zero. For every integer $n \geq 0$ we consider the following subsets of $\mathbf{R}$ :

$$
\begin{aligned}
\mathcal{T}_{n}(k) & :=\{\operatorname{lct}(X, Y) \mid X \text { smooth variety over } k \text { of dimension } n, \varnothing \neq Y \subseteq X\} \\
\mathcal{T}_{n}^{\text {pol }}(k) & :=\left\{\operatorname{lct}_{0}(\mathfrak{a}) \mid \mathfrak{a} \subseteq\left(x_{1}, \ldots, x_{n}\right) k\left[x_{1}, \ldots, x_{n}\right]\right\} \\
\mathcal{T}_{n}^{\operatorname{ser}}(k) & :=\left\{\operatorname{lct}(\widetilde{\mathfrak{a}}) \mid \tilde{\mathfrak{a}} \subseteq\left(x_{1}, \ldots, x_{n}\right) k \llbracket x_{1}, \ldots, x_{n} \rrbracket\right\} .
\end{aligned}
$$

Note that all these sets are contained in $\mathbf{Q}$. We also consider the set $\mathcal{T}_{n}^{\text {iso }}(k) \subseteq \mathcal{T}_{n}(k)$ of $\log$ canonical thresholds of pairs $(X, Y)$, with $X$ smooth and $n$-dimensional, $Y$ nonempty, and such that the $\log$ canonical threshold locus $\operatorname{LCT}(X, Y)$ is zero-dimensional (by convention, we put $\left.\mathcal{T}_{0}^{\text {iso }}(k)=\{0\}\right)$.

It is clear that we have $\mathcal{T}_{n-1}(k) \subseteq \mathcal{T}_{n}(k)$ for every $n \geq 1$ (and similar inclusions for the other sets). Indeed, this follows from the fact that $\operatorname{lct}(X, Y)=\operatorname{lct}\left(X \times \mathbf{A}^{1}, Y \times \mathbf{A}^{1}\right)$.

Before discussing some basic properties of the sets we have just introduced, we make some general remarks about log canonical thresholds of polynomials of bounded degree. We start by recalling an interpretation of the log canonical threshold in terms of jet schemes from [16]. Let us fix an algebraically closed field $k$ of characteristic zero. Recall that if $X$ is a scheme of finite type over $k$, then the $m^{\text {th }}$ jet scheme of $X$ is a scheme $X_{m}$ of finite type over $X$, such that the $k$-points of $X_{m}$ are in natural bijection with $\operatorname{Hom}\left(\operatorname{Spec} k[t] /\left(t^{m+1}\right), X\right)$. If $P \in X$ is a point, then we denote by $X_{m, P}$ the fiber of $X_{m}$ over $P$. It is proved in Corollary 3.6 in loc. cit. that if $X$ is smooth and $Y \hookrightarrow X$ is a closed subscheme containing the point $P \in X$, then

$$
\operatorname{lct}_{P}(X, Y)=\operatorname{dim}(X)-\sup _{m \geq 0} \frac{\operatorname{dim}\left(Y_{m, P}\right)}{m+1} .
$$

An important remark for our applications is the fact that if we have a family of subschemes $\mathcal{Y} \hookrightarrow S \times \mathbb{A}^{N}$ parametrized by $S$ and defined over a subfield $k_{0}$ of $k$, then we have a closed subscheme $(\mathcal{Y} / S)_{m} \hookrightarrow S \times\left(\mathbb{A}^{N}\right)_{m}$ defined over $k_{0}$, whose fiber over a point $t \in S$ is the $m^{\text {th }}$ jet scheme of the fiber of $\mathcal{Y}$ over $t$.

We now turn to describing the set of ideals generated in bounded degree and having constant $\log$ canonical threshold. Suppose that $L$ is an algebraically closed field containing our base field $k$. Since there are $r:=\left(\begin{array}{c}n+d \\ n\end{array}\right)-1$ monomials of positive degree $\leq d$ in $L\left[x_{1}, \ldots, x_{n}\right]$, it follows that every ideal $\mathfrak{b} \subseteq\left(x_{1}, \ldots, x_{n}\right) L\left[x_{1}, \ldots, x_{n}\right]$ generated in degree $\leq d$ can be generated by $r$ linear combinations of these monomials. Hence we can find a parameter space $W=\mathbf{A}^{r^{2}}$ such that for every such $L$, we have a functorial surjective map

$$
\varphi_{L}: W(L) \rightarrow\left\{\mathfrak{b} \subseteq\left(x_{1}, \ldots, x_{n}\right) L\left[x_{1}, \ldots, x_{n}\right] \mid \mathfrak{b} \text { generated in degree } \leq d\right\} .
$$

Here we denote by $W(L)$ the set of $L$-valued points of $W$. There is also a closed subscheme $\mathcal{Y} \hookrightarrow W \times \mathbf{A}^{n}$ defined over $\mathbf{Q}$, such that the fiber of $\mathcal{Y}$ over $u \in W(L)$ is the subscheme defined by the ideal $\varphi_{L}(u)$.

For every $L$ as above, and for every $\lambda \in \mathbf{R}$, consider the set

$$
W(L)_{<\lambda}:=\left\{u \in W(L) \mid \operatorname{lct}_{0}\left(\varphi_{L}(u)\right)<\lambda\right\} .
$$

$4^{\text {e }}$ SÉRIE - TOME $42-2009-\mathrm{N}^{\mathrm{o}} 3$ 
This is a closed subset of $W(L)$ by the semicontinuity property of log canonical thresholds (see Corollary 9.5.39 in [13], or Theorem 4.9 in [16]). On the other hand, consider for every $i \in \mathbf{N}$ the closed subset $S_{i}$ of $W$ such that for every $L$, the points in $S_{i}(L)$ correspond to those $u \in W(L)$ having the fiber over 0 of the $i^{\text {th }}$ jet scheme of $V\left(\varphi_{L}(u)\right)$ of dimension strictly greater than $(i+1)(n-\lambda)$ (the fact that $S_{i}$ is closed in $W$ follows using the $\mathbf{G}_{m}$-action on jet schemes, see Proposition 2.3 in [16]). We deduce from (8) that for every $L$, we have $W(L)_{<\lambda}=\bigcup_{i \geq 0} S_{i}(L)$. A key point is that each $S_{i}$ is defined over our base field $k$ (in fact, over the algebraic closure $\overline{\mathbf{Q}}$ of $\mathbf{Q}$ ).

Let us now fix an extension $L$ of $k$ that is uncountable (and algebraically closed). Since $\bigcup_{i \geq 0} S_{i}(L)$ is closed, it follows that there is $s \in \mathbf{N}$ such that $S_{i} \subseteq \cup_{j=0}^{s} S_{j}$ for every $i$. If we put $W_{<\lambda}:=\cup_{j=0}^{s} S_{j}$, then we see that this is a closed algebraic subset of $W$ defined over $k$, such that for every extension $K$ of $k$, the points of $W_{<\lambda}(K) \subseteq W(K)$ correspond to $W(K)_{<\lambda}$. Note that there are only finitely many distinct $W_{<\lambda}$ as $\lambda$ varies in $\mathbf{R}$ : this follows from the fact that over $k$ there are only finitely many possible log canonical thresholds corresponding to ideals parametrized by a scheme of finite type over $k$ (see, for example, Lemma 4.8 in [16]). The above discussion implies that the same will hold over every field:

Proposition 3.1. - For every $d$, the set of log canonical thresholds of ideals of $k\left[x_{1}, \ldots, x_{n}\right]$ generated by polynomials of degree $\leq d$ is finite and independent of the ground field $k$. In particular, the set $\mathcal{T}_{n}^{\text {pol }}(k)$ is independent of the (algebraically closed) field $k$.

Proof. - After a linear change of coordinates, we see that it is enough to consider log canonical thresholds at the origin. In this case, it is enough to run the above argument with $k$ replaced by $\overline{\mathbf{Q}}$. We have already mentioned that there are only finitely many possible log canonical thresholds. Moreover, $\lambda$ really is such a log canonical threshold if and only if $W_{<\lambda} \neq W_{<\lambda^{\prime}}$ for every $\lambda^{\prime}>\lambda$. This condition is independent of the ground field, hence our assertion.

REMARK 3.2. - Of course, the above proposition can be also proved using the description of log canonical thresholds in terms of log resolutions. However, we decided to give the above argument using jet schemes, since in the next section we will need to make use of this setting anyway.

Proposition 3.3. - For every $n$ and every $k$, we have $\mathcal{T}_{n}(k)=\mathcal{T}_{n}^{\mathrm{pol}}(k)=\mathcal{T}_{n}^{\operatorname{ser}}(k)$.

Proof. - The inclusion $\mathcal{T}_{n}^{\text {pol }}(k) \subseteq \mathcal{T}_{n}(k)$ is trivial, while $\mathcal{T}_{n}^{\text {ser }}(k) \subseteq \mathcal{T}_{n}^{\text {pol }}(k)$ follows from Proposition 2.11 and Proposition 3.1. On the other hand, we have $\mathcal{T}_{n}(k) \subseteq \mathcal{T}_{n}^{\text {ser }}(k)$ by Proposition 2.7: if $c=\operatorname{lct}(\mathfrak{a})$, where $\mathfrak{a}$ is an ideal on $X$, and if $p \in \operatorname{LCT}(\mathfrak{a})$, then $c=\operatorname{lct}_{p}(\mathfrak{a})=$ $\operatorname{lct}\left(\mathfrak{a} \cdot \widehat{\mathcal{O}_{X, p}}\right)$. Since $\operatorname{dim}(X)=n$, we have $\widehat{\mathcal{O}_{X, p}} \simeq k \llbracket x_{1}, \ldots, x_{n} \rrbracket$, hence our assertion.

In light of Propositions 3.1 and 3.3, from now on we simply write $\mathcal{T}_{n}$ for either of $\mathcal{T}_{n}(k)$, $\mathcal{T}_{n}^{\text {pol }}(k)$, or $\mathcal{T}_{n}^{\text {ser }}(k)$.

Proposition 3.4. - For every $n \geq 1$ we have

i) $\mathcal{T}_{n}^{\text {iso }}(k)$ is independent of $k$ (hence we simply write $\mathcal{T}_{n}^{\text {iso }}$ instead of $\mathcal{T}_{n}^{\text {iso }}(k)$ ).

ii) $\mathcal{T}_{n}=\mathcal{T}_{n-1} \cup \mathcal{T}_{n}^{\text {iso }}$.

iii) $\mathcal{T}_{n}=\bigcup_{i=0}^{n} \mathcal{T}_{i}^{\text {iso }}$. 
Proof. - The proof of i) is similar to that of Proposition 3.1, so we just describe the required modifications. If $k \subseteq K$ are algebraically closed fields, then the inclusion $\mathcal{T}_{n}^{\text {iso }}(k) \subseteq \mathcal{T}_{n}^{\text {iso }}(K)$ is clear: it is enough to use Proposition 2.9.

For the reverse inclusion, note that if $c \in \mathcal{T}_{n}^{\text {iso }}(K)$, then by Proposition 2.11 we can write $c=\operatorname{lct}_{0}(\mathfrak{a})$ for an ideal $\mathfrak{a} \subseteq\left(x_{1}, \ldots, x_{n}\right) \cdot K\left[x_{1}, \ldots, x_{n}\right]$ cosupported at $\{0\}$. Fix $d \gg 0$ such that $x_{i}^{d} \in \mathfrak{a}$ for every $i$, and such that $\mathfrak{a}$ is generated in degree $\leq d$. We mimic the construction preceding Proposition 3.1, replacing $W=\mathbf{A}^{r^{2}}$ by the parameter space $W^{\prime}=\mathbf{A}^{(r-n)^{2}}$ defined so that $W^{\prime}(K)$ gives all ideals generated in degree $\leq d$ and containing all the $x_{i}^{d}$. We see that there is a locally closed subset $A$ of $W^{\prime}$ defined over $\overline{\mathbf{Q}}$, such that $A(K)$ corresponds to those ideals having $\log$ canonical threshold $c$. Since $A \neq \varnothing$, we have $A(k) \neq \varnothing$, hence we find a zero-dimensional ideal $\mathfrak{b} \subseteq\left(x_{1}, \ldots, x_{n}\right) \cdot k\left[x_{1}, \ldots, x_{n}\right]$ cosupported at the origin and such that $c=\operatorname{lct}(\mathfrak{b})$. We clearly have $\operatorname{LCT}(\mathfrak{b})=\{0\}$, hence $c \in \mathcal{T}_{n}^{\text {iso }}(k)$. This completes the proof of i).

For ii), fix an algebraically closed field $k$. It follows from definition that $\mathcal{T}_{n-1} \cup \mathcal{T}_{n}^{\text {iso }} \subseteq \mathcal{T}_{n}$. Suppose now that $c \in \mathcal{T}_{n} \backslash \mathcal{T}_{n}^{\text {iso }}$. We have $c=\operatorname{lct}(X, Y)$, where $X$ is an $n$-dimensional smooth variety over $k$ and $\operatorname{dim} \operatorname{LCT}(X, Y)>0$. It follows from Proposition 2.11 that $c \in \mathcal{T}_{n-1}$, hence ii). The assertion in iii) is an immediate consequence.

REMARK 3.5. - The set $\mathcal{T}_{n}^{\text {iso }}$ is dense in $\mathcal{T}_{n}$. In fact, every $c \in \mathcal{T}_{n}$ is the limit of a decreasing (possibly constant) sequence in $\mathcal{T}_{n}^{\text {iso }}$. Indeed, if $c=\operatorname{lct}_{x}(\mathfrak{a})$, for an ideal $\mathfrak{a} \subseteq \mathfrak{m}_{x}$ on the $n$-dimensional smooth variety $X$ (here $\mathfrak{m}_{x}$ is the ideal of the point $x$ ), then Proposition 2.5 shows that $\left\{\operatorname{lct}_{x}\left(\mathfrak{a}+\mathfrak{m}_{x}^{\ell}\right)\right\}_{\ell}$ is a decreasing sequence in $\mathcal{T}_{n}^{\text {iso }}$ converging to $c$.

We now define similar sets by considering only pairs $(X, Y)$, with $Y$ locally principal. More precisely, we denote by $\mathcal{H} \mathcal{T}_{n}(k)$ the set of all $\operatorname{lct}(X, Y)$, where $X$ is a smooth variety over $k$ of dimension $n$ and $Y$ is a nonempty closed subscheme locally defined by one equation. We similarly put

$$
\begin{aligned}
& \mathcal{H} \mathcal{T}_{n}^{\mathrm{pol}}(k):=\left\{\operatorname{lct}_{0}(f) \mid f \in\left(x_{1}, \ldots, x_{n}\right) k\left[x_{1}, \ldots, x_{n}\right]\right\} \\
& \mathcal{H} \mathcal{T}_{n}^{\mathrm{ser}}(k):=\left\{\operatorname{lct}(\tilde{f}) \mid \tilde{f} \in\left(x_{1}, \ldots, x_{n}\right) k \llbracket x_{1}, \ldots, x_{n} \rrbracket\right\} .
\end{aligned}
$$

We also define $\mathcal{H} \mathcal{T}_{n}^{\text {iso }}(k) \subseteq \mathcal{H} \mathcal{T}_{n}(k)$ by requiring that $\operatorname{LCT}(X, Y)$ is zero-dimensional. For future reference, we record in the following proposition some easy properties of the sets $\mathcal{T}_{n}$ and $\mathcal{H} \mathcal{T}_{n}$.

Proposition 3.6. - Given $n \geq 1$, we have

i) $\mathcal{T}_{n} \subseteq[0, n] \cap \mathbf{Q}$.

ii) $\mathcal{H} \mathcal{T}_{n}(k)=\mathcal{T}_{n}(k) \cap[0,1]$ and $\mathcal{H} \mathcal{T}_{n}^{\mathrm{pol}}(k)=\mathcal{T}_{n}^{\mathrm{pol}}(k) \cap[0,1]$.

iii) For every positive integer $m$, we have $\frac{1}{m} \cdot \mathcal{T}_{n} \subseteq \mathcal{T}_{n}$ and $\frac{1}{m} \cdot \mathcal{H} \mathcal{T}_{n}(k) \subseteq \mathcal{H} \mathcal{T}_{n}(k)$.

iv) $\mathcal{T}_{n} \subseteq n \cdot \mathcal{H} \mathcal{T}_{n}(k)$.

Proof. - All assertions are well-known. For i) one uses the fact that if $\mathfrak{a}$ is an ideal sheaf on a smooth variety $X$ vanishing at the point $P$, and if $E$ is the exceptional divisor on the blowing-up $X^{\prime}$ of $X$ at $P$, then

$$
\operatorname{lct}_{P}(\mathfrak{a}) \leq \frac{\operatorname{ord}_{E}\left(K_{X^{\prime}} / X\right)+1}{\operatorname{ord}_{E}(\mathfrak{a})}=\frac{n}{\operatorname{ord}_{E}(\mathfrak{a})} \leq n .
$$

$4^{\text {e }}$ SÉRIE - TOME $42-2009-\mathrm{N}^{\mathrm{o}} 3$ 
For the assertion in ii) we use the fact that if $\mathfrak{a} \subset \mathcal{O}_{X}$ is a proper ideal, with $X$ affine, and if $f \in \mathfrak{a}$ is a general linear combination of a set of generators, then $\mathcal{J}\left(\mathfrak{a}^{\lambda}\right)=\mathcal{J}\left(f^{\lambda}\right)$ for every $\lambda<1$ (see Prop. 9.2.28 in [13]). In particular, if $\operatorname{lct}(\mathfrak{a}) \leq 1$, then $\operatorname{lct}(\mathfrak{a})=\operatorname{lct}(f)$.

Note that iii) follows from the fact that $\operatorname{lct}\left(\mathfrak{a}^{m}\right)=\frac{1}{m} \operatorname{lct}(\mathfrak{a})$, and iv) is a consequence of i)-iii).

COROLlaRy 3.7. - For every $n$, the set $\mathcal{H T}_{n}(k)$ is independent of the algebraically closed field $k$ (hence we denote it simply by $\mathcal{H} \mathcal{T}_{n}$ ).

Proof. - The assertion follows from Proposition 3.6 ii) and the analogous property of $\mathcal{T}_{n}(k)$.

COROLLARY 3.8. - For every algebraically closed field $k$, we have

$$
\mathcal{H} \mathcal{T}_{n}(k)=\mathcal{H} \mathcal{T}_{n}^{\mathrm{pol}}(k)=\mathcal{H} \mathcal{T}_{n}^{\mathrm{ser}}(k) .
$$

Proof. - The fact that $\mathcal{H T}_{n}(k)=\mathcal{H} \mathcal{T}_{n}^{\text {pol }}(k)$ follows from Proposition 3.6 ii). The inclusion $\mathcal{H} \mathcal{T}_{n}^{\text {ser }}(k) \subseteq \mathcal{H} \mathcal{T}_{n}(k)$ follows from Propositions 3.3 and 3.6 ii), and the reverse inclusion follows from Proposition 2.7.

In light of the above two corollaries, we simply write $\mathcal{H} \mathcal{T}_{n}$ for either of the sets $\mathcal{H} \mathcal{T}_{n}(k)$, $\mathcal{H} \mathcal{T}_{n}^{\text {pol }}(k)$ or $\mathcal{H} \mathcal{T}_{n}^{\text {ser }}(k)$. Note that we have $\mathcal{H} \mathcal{T}_{n}=\mathcal{T}_{n} \cap[0,1]$.

REMARK 3.9. - We also have $\mathcal{H} \mathcal{T}_{n}^{\text {iso }}(k)=\mathcal{T}_{n}^{\text {iso }}(k) \cap[0,1)$ for $n \geq 2$. Indeed, note first that for every locally principal ideal sheaf $\mathfrak{a}$, we have $\mathcal{J}(\mathfrak{a})=\mathfrak{a}$, hence $1 \notin \mathcal{H} \mathcal{T}_{n}^{\text {iso }}(k)$. On the other hand, suppose that we have $\operatorname{lct}(\mathfrak{a})<1$, where $\mathfrak{a} \subset \mathcal{O}_{X}$ is a proper ideal sheaf, with $X$ affine, nonsingular and $n$-dimensional, and let $f \in \mathfrak{a}$ be a general linear combination of a system of generators of $\mathfrak{a}$. Since $\mathcal{J}\left(\mathfrak{a}^{\lambda}\right)=\mathcal{J}\left(f^{\lambda}\right)$ for every $\lambda<1$, we see that $\operatorname{lct}(f)=\operatorname{lct}(\mathfrak{a})$ and $\operatorname{LCT}(f)=\operatorname{LCT}(\mathfrak{a})$.

Together with Proposition 3.4, this implies that $\mathcal{H T}_{n}^{\text {iso }}(k)$ is independent of $k$, and therefore we simply write $\mathcal{H} \mathcal{T}_{n}^{\text {iso }}$. We also deduce that $\mathcal{H} \mathcal{T}_{n}=\mathcal{H} \mathcal{T}_{n-1} \cup \mathcal{H} \mathcal{T}_{n}^{\text {iso }}$ for every $n \geq 1$.

\section{Limits of log canonical thresholds via ultrafilter constructions}

Our main goal in this section is to prove Theorems 1.3 and 1.5. Recall first some terminology. If $T$ is a subset of $\mathbf{R}$, then an element $r \in \mathbf{R}$ is said to be a point of accumulation (or accumulation point) of $T$ if $T \cap(r-\varepsilon, r+\varepsilon) \backslash\{r\} \neq \varnothing$ for every $\varepsilon>0$. We say that $r$ is a point of accumulation from above (resp. from below) of $T$ if $T \cap(r, r+\varepsilon) \neq \varnothing$ (resp. $T \cap(r-\varepsilon, r) \neq \varnothing)$ for every $\varepsilon>0$.

The key arguments in this section are based on ultrafilter constructions. For basic definitions and properties, we refer to [10]. In the following we fix a non-principal ultrafilter $\mathcal{U}$ on the set of nonnegative integers $\mathbf{N}$. We say that a property $\mathcal{P}(m)$ holds for almost all $m \in \mathbf{N}$ if the set $\{m \in \mathbf{N} \mid \mathcal{P}(m)$ holds $\}$ belongs to $\mathcal{U}$.

The ultraproduct (with respect to the ultrafilter $\mathcal{U}$ ) of a sequence of sets $\left\{A_{m}\right\}_{m \in \mathbf{N}}$ will be denoted by $\left[A_{m}\right]$. Recall that this consists of equivalence classes of sequences $\left(a_{m}\right)_{m}$, where $\left(a_{m}\right)_{m} \sim\left(b_{m}\right)_{m}$ if $a_{m}=b_{m}$ for almost all $m$. The class of a sequence of elements $\left(a_{m}\right)_{m}$ in $\left[A_{m}\right]$ will be denoted by $\left[a_{m}\right]$. 
Given a sequence of functions $f_{m}: A_{m} \rightarrow B_{m}$, we denote by $\left[f_{m}\right]:\left[A_{m}\right] \rightarrow\left[B_{m}\right]$ the function that takes $\left[a_{m}\right]$ to $\left[f\left(a_{m}\right)\right]$. If $A_{m}=A$ for all $m$, then the corresponding ultraproduct is simply denoted by ${ }^{*} A$, and called the non-standard extension of $A$. Note that there is an injective map $A \hookrightarrow{ }^{*} A$ that takes $a$ to the class of $(a, a, \ldots)$. Similarly, a function $u: A \rightarrow B$ has a non-standard extension ${ }^{*} u:{ }^{*} A \rightarrow{ }^{*} B$. If $\left(A_{m}\right)_{m}$ is a sequence of sets, and if $B_{m} \subseteq A_{m}$ for every $m$ (in fact, it is enough to have this inclusion for almost all $m$ ), then $\left[B_{m}\right]$ can be considered a subset of $\left[A_{m}\right]$. The subsets of $\left[A_{m}\right]$ that arise in this way are called internal. Similarly, if $\left(A_{m}\right)_{m}$ and $\left(B_{m}\right)_{m}$ are sequences of sets, then an internal function $f:\left[A_{m}\right] \longrightarrow\left[B_{m}\right]$ is a function of the form $f=\left[f_{m}\right]$ for suitable $f_{m}: A_{m} \rightarrow B_{m}$.

As a general principle one observes that if $A$ has an algebraic structure, then ${ }^{*} A$ has a similar structure, too. For example, ${ }^{*} \mathbf{R}$ is an ordered field, and if $k$ is an algebraically closed field, then ${ }^{*} k$ is an algebraically closed field, too. The operations are defined component-wise, for example $\left[a_{m}\right]+\left[b_{m}\right]=\left[a_{m}+b_{m}\right]$.

We now turn to the case that will be of particular interest to us. Suppose that $k$ is a field, and that we have a sequence of polynomials $f_{m} \in k\left[x_{1}, \ldots, x_{n}\right]$. We can view any polynomial $g \in k\left[x_{1}, \ldots, x_{n}\right]$ as a function $\mathbf{N}^{n} \rightarrow k$ given by sending the tuple $\left(m_{1}, \ldots, m_{n}\right)$ to the coefficient of the monomial $x_{1}^{m_{1}} \cdots x_{n}^{m_{n}}$ in $g$. The sequence $\left(f_{m}\right)_{m}$ gives an internal polynomial $F=\left[f_{m}\right] \in{ }^{*}\left(k\left[x_{1}, \ldots, x_{n}\right]\right)$, that we can view as a function ${ }^{*}\left(\mathbf{N}^{n}\right) \rightarrow{ }^{*} k$. Since we have a natural inclusion $\mathbf{N}^{n} \subset{ }^{*}\left(\mathbf{N}^{n}\right)$, we can restrict $F$ to $\mathbf{N}^{n}$ to get a formal power series $\tilde{f} \in{ }^{*} k \llbracket x_{1}, \ldots, x_{n} \rrbracket$. Hence we have the following natural maps

$$
k\left[x_{1}, \ldots, x_{n}\right] \hookrightarrow^{*}\left(k\left[x_{1}, \ldots, x_{n}\right]\right) \stackrel{\rho}{\rightarrow} * \llbracket \llbracket x_{1}, \ldots, x_{n} \rrbracket .
$$

Note that we have a natural inclusion ${ }^{*} k\left[x_{1}, \ldots, x_{n}\right] \subset \subset^{*}\left(k\left[x_{1}, \ldots, x_{n}\right]\right)$ such that the restriction of $\rho$ to ${ }^{*} k\left[x_{1}, \ldots, x_{n}\right]$ is the usual inclusion of the polynomial ring in the formal power series ring. We also remark that if $f_{m}(0)=0$ for almost all $m$, then $\widetilde{f}$ lies in the maximal ideal, i.e. $\widetilde{f}(0)=0$.

The above construction can be generalized to ideals. Given a sequence of ideals $\mathfrak{a}_{m} \subseteq k\left[x_{1}, \ldots, x_{n}\right]$, we get the internal ideal $A=\left[\mathfrak{a}_{m}\right] \subseteq{ }^{*}\left(k\left[x_{1}, \ldots, x_{n}\right]\right)$, with

$$
A=\left\{F=\left[f_{m}\right] \mid f_{m} \in \mathfrak{a}_{m} \text { for almost all } m\right\} .
$$

Note that $A$ is indeed an ideal in the ring of internal polynomials ${ }^{*}\left(k\left[x_{1}, \ldots, x_{n}\right]\right)$. We denote by $\widetilde{\mathfrak{a}} \subseteq{ }^{*} k \llbracket x_{1}, \ldots, x_{n} \rrbracket$ the ideal generated by $\rho(A)$, that is, the ideal generated by the restrictions of the elements in $A$ to $\mathbf{N}^{n}$. We will refer to $\widetilde{\mathfrak{a}}$ as the ideal of power series associated to $A$.

We say that an internal polynomial $G=\left[g_{m}\right] \in{ }^{*}\left(k\left[x_{1}, \ldots, x_{n}\right]\right)$ has bounded degree if there exists an integer $d$ such that $\operatorname{deg}\left(g_{m}\right) \leq d$ for almost all $m$. Note that every internal polynomial $G$ of bounded degree can be represented as $G=\left[g_{m}^{\prime}\right]$, where $g_{m}^{\prime}$ are polynomials of degree uniformly bounded by some $d$, and thus it can be viewed as a function ${ }^{*}\left(\mathbf{N}^{n}\right) \rightarrow{ }^{*} k$ that is zero away from a finite subset of $\mathbf{N}^{n}$. As such, $G$ can be naturally identified with a polynomial $g \in{ }^{*} k\left[x_{1}, \ldots, x_{n}\right]$ (of course, $g$ is independent of the choice of the polynomials $g_{m}^{\prime}$ chosen to represent $G$ ).

An internal ideal $B=\left[\mathfrak{b}_{m}\right] \subseteq{ }^{*}\left(k\left[x_{1}, \ldots, x_{n}\right]\right)$ is generated in degree $\leq d \in \mathbf{N}$ if $\mathfrak{b}_{m}$ can be generated in degree $\leq d$ for almost all $m$. Since $\operatorname{dim}_{k} k\left[x_{1}, \ldots, x_{n}\right]_{\leq d}=\left(\begin{array}{c}d+n \\ n\end{array}\right)=r+1$, 
we deduce that if $B$ is generated in degree $\leq d$, then we may assume that every $\mathfrak{b}_{m}$ is generated by a set of $r+1$ polynomials of degree $\leq d$. Thus we can write $\mathfrak{b}_{m}=\left(g_{0, m}, \ldots, g_{r, m}\right)$, with $\operatorname{deg} g_{i, m} \leq d$. Then we see that $B$ is generated by the internal polynomials $G_{i}=\left[g_{i, m}\right]$ (for $0 \leq i \leq r$ ), each having degree $\leq d$. It follows that the ideal $\widetilde{\mathfrak{b}}$ of power series associated to $B$ is the extension of the ideal $\mathfrak{b} \subseteq{ }^{*} k\left[x_{1}, \ldots, x_{n}\right]$ generated by the polynomials in the ring ${ }^{*} k\left[x_{1}, \ldots, x_{n}\right]$ naturally identified with the $G_{i}$. We will refer to the ideal $\mathfrak{b}$ (which is independent of the choice of $g_{i, m}$ ), as the ideal of polynomials associated to $B$.

This applies, for instance, in the following situation. Consider the maximal ideal $\mathfrak{m}=\left(x_{1}, \ldots, x_{n}\right)$ in the polynomial ring $k\left[x_{1}, \ldots, x_{n}\right]$. If no confusion is likely to arise, we will also denote by $\mathfrak{m}$ the ideal generated by the variables in $\left.* k x_{1}, \ldots, x_{n}\right]$, and we will write $\widetilde{\mathfrak{m}}$ for the maximal ideal of ${ }^{*} k \llbracket x_{1}, \ldots, x_{n} \rrbracket$. Note that the internal ideal $M=[\mathfrak{m}]$ is equal to the ideal $\left(x_{1}, \ldots, x_{n}\right) \cdot *\left(k\left[x_{1}, \ldots, x_{n}\right]\right)$ generated by $x_{1}, \ldots, x_{n}$ in $*\left(k\left[x_{1}, \ldots, x_{n}\right]\right)$.

For every internal ideal $A=\left[\mathfrak{a}_{m}\right] \subseteq{ }^{*}\left(k\left[x_{1}, \ldots, x_{n}\right]\right)$ and every $d \in \mathbf{N}$, we consider the internal ideal

$$
A+M^{d}:=\left[\mathfrak{a}_{m}+\mathfrak{m}^{d}\right] \subseteq{ }^{*}\left(k\left[x_{1}, \ldots, x_{n}\right]\right) .
$$

Notice that $A+M^{d}$ is generated in degree $\leq d$. We denote by $\mathfrak{a}+\mathfrak{m}^{d} \subseteq{ }^{*} k\left[x_{1}, \ldots, x_{n}\right]$ the ideal of polynomials associated to $A+M^{d}$ (note that in this notation $\mathfrak{a}$ alone might not be defined as an ideal). If $\widetilde{\mathfrak{a}}$ and $\widetilde{\mathfrak{m}}^{d}$ are the ideals in ${ }^{*} k \llbracket x_{1}, \ldots, x_{n} \rrbracket$ associated, respectively, to $A$ and to $M^{d}$, then $\left(\mathfrak{a}+\mathfrak{m}^{d}\right) \cdot{ }^{*} k \llbracket x_{1}, \ldots, x_{n} \rrbracket=\widetilde{\mathfrak{a}}+\widetilde{\mathfrak{m}}^{d}$.

We now consider the behavior of codimension under the previous construction. Suppose that $\mathfrak{b}_{m} \subseteq k\left[x_{1}, \ldots, x_{n}\right]$, for $m \in \mathbf{N}$, is an ideal generated in degree $\leq d$, and let $\mathfrak{b} \subseteq{ }^{*} k\left[x_{1}, \ldots, x_{n}\right]$ be the ideal of polynomials associated to $\left[\mathfrak{b}_{m}\right]$. The codimension of the ideals $\mathfrak{b}_{m}$ can take only finitely many values, hence there is a unique integer $e$ such that $\operatorname{codim}\left(\mathfrak{b}_{m}\right)=e$ for almost all $m$.

Proposition 4.1. - With the above notation, we have $\operatorname{codim}(\mathfrak{b})=e$.

Proof. - We give two different proofs. Suppose first that $\mathfrak{a}=\left(f_{0}, \ldots, f_{r}\right)$ is an ideal in $k\left[x_{1}, \ldots, x_{n}\right]$ generated by $r+1$ polynomials of degree $\leq d$, where as above $r=\left(\begin{array}{c}n+d \\ n\end{array}\right)-1$. It is known that there is a first-order formula (Codim $=\mathrm{e})_{d}$ in the coefficients of $f_{0}, \ldots, f_{r}$ such that for every field $k$ and every $f_{0}, \ldots, f_{r}$ as above, we have $\operatorname{codim}(\mathfrak{a})=e$ if and only if the coefficients of the $f_{i}$ 's satisfy this formula over $k$ (see Prop. 5.1 in [17]). Recall that a firstorder formula (in the free variables $\mathbf{z}=z_{1}, \ldots, z_{N}$ ) is an expression of the form

$$
\varphi=\left(\exists \mathbf{y}_{0}\right)\left(\forall \mathbf{y}_{1}\right) \cdots\left(\exists \mathbf{y}_{s-1}\right)\left(\forall \mathbf{y}_{s}\right) \bigvee_{i<m_{1}} \bigwedge_{j<m_{2}} p_{i j}(\mathbf{z}, \mathbf{y})=0 \wedge q_{i j}(\mathbf{z}, \mathbf{y}) \neq 0,
$$

where $p_{i j}$ and $q_{i j}$ are polynomials over $\mathbf{Z}$, and the $\mathbf{y}_{j}$ are (possibly empty) tuples of variables. One says that such a formula is satisfied over $k$ (for given $z_{1}, \ldots, z_{N} \in k$ ) if we have a true statement when the $\mathbf{y}$ variables are also assumed to take values in $k$.

Suppose now that we write our ideals as $\mathfrak{b}_{m}=\left(f_{m, 0}, \ldots, f_{m, r}\right) \subseteq k\left[x_{1}, \ldots, x_{n}\right]$, such that all $f_{m, i}$ have degree $\leq d$. If $G_{i}=\left[f_{m, i}\right] \in{ }^{*} k\left[x_{1}, \ldots, x_{n}\right]$, then we have $\mathfrak{b}=\left(G_{0}, \ldots, G_{r}\right)$. We see that $\operatorname{codim}\left(G_{0}, \ldots, G_{r}\right)=e$ if and only if the coefficients of the $G_{i}$ satisfy the formula (Codim $=\mathrm{e})_{d}$ over ${ }^{*} k$. However, this is the case if and only if, for almost all $m \in \mathbf{N}$, the coefficients of the $f_{m, i}$ satisfy the formula (Codim $\left.=\mathrm{e}\right)_{d}$ over $k$. This holds since by assumption we have $\operatorname{codim}\left(\mathfrak{b}_{m}\right)=e$ for almost all $m$. 
For the benefit of the reader without experience in model theory, we give a second, more transparent proof. After replacing $k$ by a suitable extension, we may assume that $k$ is algebraically closed. We use the fact that a polynomial ring over a field is Cohen-Macaulay, hence the codimension of an ideal $\mathfrak{a}$ in such a ring is equal to the length of any maximal regular sequence contained in $\mathfrak{a}$. Furthermore, if $\operatorname{codim}(\mathfrak{a})=e$ and $\mathfrak{a}$ is generated by elements of degree $\leq d$, then any $e$ general linear combinations of these generators with coefficients in $k$ form a regular sequence. In particular, we get such a regular sequence whose elements have all degree $\leq d$.

The key ingredients in this proof are two results from [6]. The first one (Theorem 1.11 in loc. cit.) says that given $d$ and $n$, there is a bound $N=N(d, n)$ such that, for every field $K$, and every $g_{0}, g_{1}, \ldots, g_{\ell} \in K\left[x_{1}, \ldots, x_{n}\right]$, with $\operatorname{deg}\left(g_{i}\right) \leq d$ for every $i$, if $g_{0} \in\left(g_{1}, \ldots, g_{\ell}\right)$, then $g_{0}=\sum_{i=1}^{\ell} p_{i} g_{i}$, with $\operatorname{deg}\left(p_{i}\right) \leq N$ for every $i$. The second result (Theorem 1.4 in loc. cit.) has a similar flavor: given $d$ and $n$, there is a bound $N^{\prime}=N^{\prime}(d, n)$ such that, for every field $K$, and every $g_{0}, g_{1}, \ldots, g_{\ell} \in K\left[x_{1}, \ldots, x_{n}\right]$ with $\operatorname{deg}\left(g_{i}\right) \leq d$ for all $i$, the following submodule of $\left(K\left[x_{1}, \ldots, x_{n}\right]\right)^{\ell+1}$

$$
\left\{\left(q_{0}, q_{1}, \ldots, q_{\ell}\right) \in\left(K\left[x_{1}, \ldots, x_{n}\right]\right)^{\ell+1} \mid \sum_{i=0}^{\ell} q_{i} g_{i}=0\right\}
$$

is generated by elements $\left(q_{0}, \ldots, q_{\ell}\right)$ with $\operatorname{deg}\left(q_{i}\right) \leq N^{\prime}$ for $0 \leq i \leq \ell$. In particular, if $g_{0}$ is a zero-divisor modulo $\left(g_{1}, \ldots, g_{\ell}\right)$, then there are $q_{0}, q_{1}, \ldots, q_{\ell} \in K\left[x_{1}, \ldots, x_{n}\right]$ of degree $\leq N^{\prime}$ such that $q_{0} g_{0}=\sum_{i=1}^{\ell} q_{i} g_{i}$, and $q_{0} \notin\left(g_{1}, \ldots, g_{\ell}\right)$. It is worth mentioning that the proofs of these two results use in an essential way non-standard arguments.

Suppose now that we have a sequence of ideals $\mathfrak{b}_{m}$ as in the statement of the proposition. For every $m$ in the set $I:=\left\{m \in \mathbf{N} \mid \operatorname{codim}\left(\mathfrak{b}_{m}\right)=e\right\}$, let us choose $g_{m, 1}, \ldots, g_{m, e} \in \mathfrak{b}_{m}$ of degree $\leq d$, forming a regular sequence. We get $g_{j}=\left[g_{m, j}\right] \in \mathfrak{b}$ for $1 \leq j \leq e$ (we can take arbitrary $g_{m, j}$ for $m \notin I$, as these are irrelevant). We start by showing that $g_{1}, \ldots, g_{e}$ is a regular sequence in ${ }^{*} k\left[x_{1}, \ldots, x_{n}\right]$. Indeed, otherwise we can find $i \leq e$ and $h \in{ }^{*} k\left[x_{1}, \ldots, x_{n}\right] \backslash$ $\left(g_{1}, \ldots, g_{i-1}\right)$ such that $g_{i} h \in\left(g_{1}, \ldots, g_{i-1}\right)$. This implies that for almost all $m$ we have $g_{m, i} h_{m} \in\left(g_{m, 1}, \ldots, g_{m, i-1}\right)$. For almost all $m$, we know that $g_{m, 1}, \ldots, g_{m, e}$ forms a regular sequence, hence $h_{m} \in\left(g_{m, 1}, \ldots, g_{m, i-1}\right)$. Since the degrees of $h_{m}$ are also bounded above by $\operatorname{deg}(h)$, it follows from the first result we quoted from [6] that there is $N$ such that $h_{m}=\sum_{j=1}^{i-1} p_{m, j} g_{m, j}$ and $\operatorname{deg}\left(p_{m, j}\right) \leq N$ for almost all $m$. In this case $p_{j}=\left[p_{m, j}\right] \in$ ${ }^{*} k\left[x_{1}, \ldots, x_{n}\right]$ and $h=\sum_{j=1}^{i-1} p_{j} g_{j}$, a contradiction.

In order to conclude the proof, it is enough to also show that $g_{1}, \ldots, g_{e}$ is a maximal regular sequence in the ideal $\mathfrak{b}$. For this, suppose that there is $h \in \mathfrak{b}$ such that $g_{1}, \ldots, g_{e}, h$ is a regular sequence. For almost all $m$, we have $\operatorname{codim}\left(\mathfrak{b}_{m}\right)=e$, hence $h_{m}$ is a zerodivisor modulo $\left(g_{m, 1}, \ldots, g_{m, e}\right)$. By the second result we quoted from [6] we deduce that we can find $N^{\prime}$ and polynomials $q_{m, 0}, \ldots, q_{m, e} \in k\left[x_{1}, \ldots, x_{n}\right]$ of degree $\leq N^{\prime}$ such that $q_{m, 0} \notin\left(g_{m, 1}, \ldots, g_{m, e}\right)$ and $q_{m, 0} h_{m}=\sum_{i=1}^{e} q_{m, i} g_{m, i}$ for almost all $m$. If we put $q_{i}=\left[q_{m, i}\right]$, then $q_{0} h=\sum_{i=1}^{e} q_{i} g_{i}$, and our assumption on $h$ implies $q_{0} \in\left(g_{1}, \ldots, g_{e}\right)$. This contradicts the fact that $q_{m, 0} \notin\left(g_{m, 1}, \ldots, g_{m, e}\right)$ for almost all $m$, and completes our second proof. 
We now turn to $\log$ canonical thresholds. From now on $k$ is a fixed algebraically closed field of characteristic zero. The function

$$
\operatorname{lct}_{0}:\left\{\mathfrak{a} \subseteq k\left[x_{1}, \ldots, x_{n}\right] \mid \mathfrak{a} \text { ideal, } \mathfrak{a} \subseteq\left(x_{1}, \ldots, x_{n}\right)\right\} \rightarrow \mathbf{R}
$$

extends to a function ${ }^{*}{ }^{*} t_{0}$, defined for internal ideals contained in $\left(x_{1}, \ldots, x_{n}\right) \cdot{ }^{*}\left(k\left[x_{1}, \ldots, x_{n}\right]\right)$ and taking values in ${ }^{*} \mathbf{R}$. Explicitly, we have

$$
{ }^{*} \operatorname{lct}_{0}\left(\left[\mathfrak{a}_{m}\right]\right)=\left[\operatorname{lct}_{0}\left(\mathfrak{a}_{m}\right)\right] .
$$

Recall that for every bounded $u \in{ }^{*} \mathbf{R}$ there is a unique real number $\operatorname{sh}(u)$, the shadow of $u$, characterized by $|u-\operatorname{sh}(u)|<\varepsilon$ for every positive real number $\varepsilon$ (we abuse the notation by writing $|u|$, instead of $*|u|$ for $u \in{ }^{*} \mathbf{R}$ ). For a discussion of this notion, we refer to [10], §5.6. A useful fact (see Theorem 6.1 in loc. cit.) implies that if $\left\{c_{m}\right\}_{m}$ is a sequence of real numbers converging to $c$, then $\operatorname{sh}\left(\left[c_{m}\right]\right)=c$. The following is the key result that allows us to interpret limits of $\log$ canonical thresholds as $\log$ canonical thresholds of ideals of formal power series.

Proposition 4.2. - If $A=\left[\mathfrak{a}_{m}\right] \subseteq *\left(k\left[x_{1}, \ldots, x_{n}\right]\right)$ is an internal ideal contained in $\left(x_{1}, \ldots, x_{n}\right)$, and if $\widetilde{\mathfrak{a}} \subseteq{ }^{*} k \llbracket x_{1}, \ldots, x_{n} \rrbracket$ is the ideal of power series associated to $A$, then

$$
\operatorname{sh}\left({ }^{*} \operatorname{lct}_{0}(A)\right)=\operatorname{lct}(\widetilde{\mathfrak{a}}) .
$$

We first prove the following lemma.

Lemma 4.3. - If $B=\left[\mathfrak{b}_{m}\right] \subseteq{ }^{*}\left(k\left[x_{1}, \ldots, x_{n}\right]\right)$ is an internal ideal contained in $\left(x_{1}, \ldots, x_{n}\right)$. ${ }^{*} k\left[x_{1}, \ldots, x_{n}\right]$ and generated in degree $\leq d$, and if $\mathfrak{b} \subseteq{ }^{*} k\left[x_{1}, \ldots, x_{n}\right]$ is the ideal of polynomials associated to $B$, then

$$
{ }^{*} \operatorname{lct}_{0}(B)=\operatorname{lct}_{0}(\mathfrak{b})
$$

(where $\mathbf{R}$ is considered as a subset of ${ }^{*} \mathbf{R}$ in the usual way).

Proof. - We can assume that each $\mathfrak{b}_{m}$ is contained in $\mathfrak{m}$ and is generated in degree $\leq d$. We have seen in the previous section that there is an affine space $W=\mathbb{A}^{r^{2}}$, where $r=\left(\begin{array}{c}d+n \\ n\end{array}\right)-1$, such that for every field extension $k \subseteq L$, with $L$ algebraically closed, we have a functorial surjective map

$$
\varphi_{L}: W(L) \rightarrow\left\{\mathfrak{b} \subseteq\left(x_{1}, \ldots, x_{n}\right) L\left[x_{1}, \ldots, x_{n}\right] \mid \mathfrak{b} \text { generated in degree } \leq d\right\} .
$$

Moreover, we have seen that $W$ can be written as the disjoint union of finitely many locally closed subsets $W_{\lambda_{i}}$ (with $\lambda_{i} \in \mathbf{R}$ ) defined over $k$, such that for every $L$

$$
W_{\lambda_{i}}(L)=\left\{u \in W(L) \mid \operatorname{lct}_{0}\left(\varphi_{L}(u)\right)=\lambda_{i}\right\} .
$$

Therefore there is $\lambda$ such that $\mathfrak{b}_{m} \in W_{\lambda}$ for almost all $m$, hence ${ }^{*} \operatorname{lct}_{0}(B)=\lambda$.

If $L$ is as above, and if $\mathfrak{a} \subseteq\left(x_{1}, \ldots, x_{n}\right) L\left[x_{1}, \ldots, x_{n}\right]$, let us denote by $\mathfrak{a}^{\langle i\rangle}$ the ideal defining the $i^{\text {th }}$ jet scheme of the subscheme of $\mathbf{A}_{L}^{n}$ defined by $\mathfrak{a}$. This is an ideal in the polynomial $\operatorname{ring} R_{i}:=L\left[x_{j}^{(\ell)} \mid 1 \leq j \leq n, 0 \leq \ell \leq i\right]$ (where we identify $x_{j}^{(0)}$ with $x_{j}$ ). If $\mathfrak{a}$ is generated by $\left\{f_{\alpha}\right\}_{\alpha}$, then $\mathfrak{a}^{\langle i\rangle}$ is generated by $\left\{f_{\alpha}, f_{\alpha}^{\prime}, \ldots, f_{\alpha}^{(i)}\right\}_{\alpha}$, where $f^{(q)}=D^{q}(f)$, with $D$ being a derivation of $R_{i}$ taking $x_{j}^{(\ell)}$ to $x_{j}^{(\ell+1)}$. We refer to [9], $\S 3$, for this description by equations of the jet schemes. We denote by $\overline{\mathfrak{a}^{\langle i\rangle}} \subseteq R_{i} /\left(x_{1}, \ldots, x_{n}\right)$ the ideal defining the fiber over 0 in the above jet scheme. 
It follows from the above description that in our setup the ideal

$$
\overline{\mathfrak{b}^{\langle i\rangle}} \subseteq{ }^{*} k\left[x_{j}^{(1)}, \ldots, x_{j}^{(i)} \mid 1 \leq j \leq n\right]
$$

is the ideal of polynomials associated to the internal ideal $\left[\overline{\mathfrak{b}_{m}^{\langle i\rangle}}\right]$. Note that this is an internal ideal generated in degree $\leq d$. In particular, it follows from Proposition 4.1 that $\operatorname{codim}\left(\overline{\mathfrak{b}^{\langle i\rangle}}\right)=e_{i}$, where $e_{i}$ is the unique integer such that $\operatorname{codim}\left(\overline{\mathfrak{b}_{m}^{\langle i\rangle}}\right)=e_{i}$ for almost all $m$.

On the other hand, we have seen in $\S 3$ that there is a positive integer $N$ such that for every algebraically closed field extension $L$ of $k$, and every ideal $\mathfrak{a} \subseteq\left(x_{1}, \ldots, x_{n}\right) L\left[x_{1}, \ldots, x_{n}\right]$ that is generated in degree $\leq d$, we have $\operatorname{lct}_{0}(\mathfrak{a})=\lambda$ if and only if codim $\left(\overline{\mathfrak{a}^{\langle i\rangle}}\right)$ satisfies suitable inequalities for every $i \leq N$. Indeed, note that we have only finitely many possible log canonical thresholds for these ideals, hence given such a $\log$ canonical threshold $\lambda$, we can find $\lambda^{\prime}>\lambda$ such that there is no possible log canonical threshold in $\left(\lambda, \lambda^{\prime}\right)$. We can then choose $N$ such that the condition $\operatorname{lct}_{0}(\mathfrak{a}) \geq \lambda$ is equivalent to $\operatorname{codim}(\overline{\mathfrak{a}\langle i\rangle}) \geq(n-\lambda)(i+1)$ for every $i \leq N$, whereas the condition $\operatorname{lct}_{0}(\mathfrak{a}) \leq \lambda$ is equivalent to $\operatorname{codim}\left(\overline{\mathfrak{a}^{\langle i\rangle}}\right) \leq\left(n-\lambda^{\prime}\right)(i+1)$ for some $i \leq N$.

It follows that if these inequalities are satisfied by $\operatorname{codim}\left(\overline{\mathfrak{b}_{m}^{\langle i\rangle}}\right)$ for almost all $m$, then they are satisfied also by $\operatorname{codim}\left(\overline{\mathfrak{b}^{\langle i\rangle}}\right)$. We conclude that $\operatorname{lct}_{0}(\mathfrak{b})=\lambda$, as required.

Proof of Proposition 4.2. - For every fixed $d \geq 1$, we consider the internal ideal $A+M^{d}=\left[\mathfrak{a}_{m}+\mathfrak{m}^{d}\right]$. Note that this internal ideal is generated in degree $\leq d$. Let

$$
\mathfrak{a}+\mathfrak{m}^{d} \subseteq{ }^{*} k\left[x_{1}, \ldots, x_{n}\right] \quad \text { and } \quad \tilde{\mathfrak{a}}+\widetilde{\mathfrak{m}}^{d} \subseteq{ }^{*} k \llbracket x_{1}, \ldots, x_{n} \rrbracket
$$

denote, respectively, the ideal of polynomials and the ideal of power series that are associated to $A+M^{d}$. Since $\widetilde{\mathfrak{a}}+\widetilde{\mathfrak{m}}^{d}=\left(\mathfrak{a}+\mathfrak{m}^{d}\right) \cdot{ }^{*} k \llbracket x_{1}, \ldots, x_{n} \rrbracket$, we have

$$
\operatorname{lct}_{0}\left(\mathfrak{a}+\mathfrak{m}^{d}\right)=\operatorname{lct}\left(\widetilde{\mathfrak{a}}+\widetilde{\mathfrak{m}}^{d}\right)
$$

by Proposition 2.7. Applying Corollary 2.10, we get

$$
\left|\operatorname{lct}(\widetilde{\mathfrak{a}})-\operatorname{lct}\left(\widetilde{\mathfrak{a}}+\widetilde{\mathfrak{m}}^{d}\right)\right| \leq n / d
$$

for every $d \geq 1$, and similarly

$$
\left|{ }^{*} \operatorname{lct}_{0}(A)-{ }^{*} \operatorname{lct}_{0}\left(A+M^{d}\right)\right|=\left[\left|\operatorname{lct}_{0}\left(\mathfrak{a}_{m}\right)-\operatorname{lct}_{0}\left(\mathfrak{a}_{m}+\mathfrak{m}^{d}\right)\right|\right] \leq n / d .
$$

On the other hand, ${ }^{*} \operatorname{lct}_{0}\left(A+M^{d}\right)=\operatorname{lct}_{0}\left(\mathfrak{a}+\mathfrak{m}^{d}\right)$ for any $d$ by Lemma 4.3. Therefore

$$
\left|\operatorname{lct}(\widetilde{\mathfrak{a}})-{ }^{*} \operatorname{lct}_{0}(A)\right| \leq 2 n / d \text {. }
$$

As this holds for every $d$, we get the assertion in the proposition.

REMARK 4.4. - One can carry a construction analogous to the one considered in this section when starting with a sequence of power series $h_{m} \in k \llbracket x_{1}, \ldots, x_{n} \rrbracket$ rather than a sequence of polynomials. This produces an internal power series $H=\left[h_{m}\right] \in{ }^{*}\left(k \llbracket x_{1}, \ldots, x_{n} \rrbracket\right)$, and thus, after truncation of the unbounded degree terms, a power series $\widetilde{h} \in{ }^{*} k \llbracket x_{1}, \ldots, x_{n} \rrbracket$. Similarly, starting with a sequence of ideals $\mathfrak{a}_{m} \subseteq k \llbracket x_{1}, \ldots, x_{n} \rrbracket$, we get an ideal $A$ in ${ }^{*}\left(k \llbracket x_{1}, \ldots, x_{n} \rrbracket\right)$, and after truncation, an ideal $\tilde{\mathfrak{a}} \subseteq{ }^{*} k \llbracket x_{1}, \ldots, x_{n} \rrbracket$. All previous results have analogues in this setting, the proofs being the same. For example, we again have $\operatorname{sh}\left({ }^{*} \operatorname{lct}_{0}(A)\right)=\operatorname{lct}(\widetilde{\mathfrak{a}})$. 
We are now ready to prove our main results stated in the introduction.

Proof of Theorem 1.3. - Consider a sequence $\left\{c_{m}\right\}_{m}$ with $c_{m} \in \mathcal{T}_{n}$ for all $m$, and with $\lim _{m \rightarrow \infty} c_{m}=c$. Fix an algebraically closed field $k$ of characteristic zero. By Proposition 3.3 we can find ideals $\mathfrak{a}_{m} \subseteq\left(x_{1}, \ldots, x_{n}\right) k\left[x_{1}, \ldots, x_{n}\right]$ such that $c_{m}=\operatorname{lct}_{0}\left(\mathfrak{a}_{m}\right)$. Let $A=\left[\mathfrak{a}_{m}\right]$, and let $\tilde{\mathfrak{a}} \subseteq{ }^{*} k \llbracket x_{1}, \ldots, x_{n} \rrbracket$ be the ideal of formal power series associated to $A$. It follows from Proposition 4.2 that

$$
\operatorname{sh}\left(\left[c_{m}\right]\right)=\operatorname{sh}\left({ }^{*} \operatorname{lct}_{0}(A)\right)=\operatorname{lct}(\widetilde{\mathfrak{a}}) .
$$

On the other hand, since $\left\{c_{m}\right\}_{m}$ converges to $c$, we have $\operatorname{sh}\left(\left[c_{m}\right]\right)=c$. We conclude that $c \in \mathcal{T}_{n}^{\text {ser }}$, and therefore $c \in \mathcal{T}_{n}$, by Proposition 3.3.

Proof of Theorem 1.5. - The fact that every element of $\mathcal{T}_{n-1}$ is a point of accumulation from above for $\mathcal{T}_{n}$ is well-known. It follows from the fact that if $c=\operatorname{lct}(\mathfrak{a})$, for some ideal sheaf $\mathfrak{a}$ on a nonsingular $(n-1)$-dimensional variety $X$, then $c+\frac{1}{m}=\operatorname{lct}\left(\mathfrak{a}+\left(t^{m}\right)\right)$, where $\mathfrak{a}+\left(t^{m}\right)$ is an ideal on $X \times \mathbb{A}^{1}, t$ denoting the coordinate on $\mathbb{A}^{1}$ (see, for example, Proposition 1.20 in [8]).

Suppose now that we have a strictly decreasing sequence $\left\{c_{m}\right\}_{m \in \mathbf{N}}$ in $\mathcal{T}_{n}$, and let $c=\lim _{m \rightarrow \infty} c_{m}$. Fix an algebraically closed field $k$ of characteristic zero. By Proposition 3.3 we can find ideals $\mathfrak{a}_{m} \subseteq\left(x_{1}, \ldots, x_{m}\right) k\left[x_{1}, \ldots, x_{n}\right]$ such that $c_{m}=\operatorname{lct}_{0}\left(\mathfrak{a}_{m}\right)$. Let $A=\left[\mathfrak{a}_{m}\right]$, and let $\widetilde{\mathfrak{a}} \subseteq{ }^{*} k \llbracket x_{1}, \ldots, x_{n} \rrbracket$ be the ideal of formal power series associated to $A$. As in the proof of Theorem 1.3, we deduce that $c=\operatorname{lct}(\widetilde{\mathfrak{a}})$.

As usual, we put $\mathfrak{m}=\left(x_{1}, \ldots, x_{n}\right) \cdot k\left[x_{1}, \ldots, x_{n}\right]$ and $\widetilde{\mathfrak{m}}=\left(x_{1}, \ldots, x_{n}\right) \cdot{ }^{*} \llbracket \llbracket x_{1}, \ldots, x_{n} \rrbracket$. In order to complete the proof, it is enough to show that

$$
\operatorname{lct}\left(\widetilde{\mathfrak{a}}+\widetilde{\mathfrak{m}}^{d}\right)>\operatorname{lct}(\widetilde{\mathfrak{a}})
$$

for every $d \geq 1$. Indeed, this implies by Proposition 2.5 that the locus $\operatorname{LCT}(\widetilde{\mathfrak{a}})$ is positive dimensional, hence $c \in \mathcal{T}_{n-1}$ by Proposition 2.11.

Fix now $d \geq 1$, and consider the ideals $\mathfrak{a}_{m}+\mathfrak{m}^{d} \subseteq k\left[x_{1}, \ldots, x_{n}\right]$. Note that the internal ideal $\left[\mathfrak{a}_{m}+\mathfrak{m}^{\bar{d}}\right]$ is equal to $A+M^{d}$, where $M=\left(x_{1}, \ldots, x_{n}\right) \cdot *\left(k\left[x_{1}, \ldots, x_{n}\right]\right)$. Therefore the ideal of formal power series associated to $\left[\mathfrak{a}_{m}+\mathfrak{m}^{d}\right]$ is $\widetilde{\mathfrak{a}}+\widetilde{\mathfrak{m}}^{d}$. Since the internal ideal $\left[\mathfrak{a}_{m}+\mathfrak{m}^{d}\right]$ is generated in degree $\leq d$, it follows from Proposition 3.1 that there is $\lambda_{d} \in \mathbf{R}$ such that $\operatorname{lct}_{0}\left(\mathfrak{a}_{m}+\mathfrak{m}^{d}\right)=\lambda_{d}$ for almost all $m$, and hence we obtain lct $\left(\widetilde{\mathfrak{a}}+\widetilde{\mathfrak{m}}^{d}\right)=\lambda_{d}$ by Proposition 4.2.

On the other hand, for every $m$ we have $\operatorname{lct}_{0}\left(\mathfrak{a}_{m}+\mathfrak{m}^{d}\right) \geq \operatorname{lct}_{0}\left(\mathfrak{a}_{m}\right)=c_{m}>c$, hence $\lambda_{d}>c$. This shows (9) and completes the proof of the theorem.

REMARK 4.5. - Since $\mathcal{H} \mathcal{T}_{n}=\mathcal{T}_{n} \cap[0,1]$ we immediately obtain variants of Theorems 1.3 and 1.5 for $\log$ canonical thresholds of hypersurfaces: each set $\mathcal{H} \mathcal{T}_{n}$ is closed, and the set of accumulation points from above of $\mathcal{H} \mathcal{T}_{n}$ is $\mathcal{H} \mathcal{T}_{n-1} \backslash\{1\}$. 


\section{Comments on the ACC Conjecture}

By Proposition 3.6, we have $\mathcal{H} \mathcal{T}_{n} \subseteq \mathcal{T}_{n} \subseteq n \cdot \mathcal{H} \mathcal{T}_{n}$. This implies that Conjecture 1.1 holds for $n$ if and only if $\mathcal{H} \mathcal{T}_{n}$ has no points of accumulation from below. We now show that this holds for every $n$ if and only if for every $n$ there is no strictly increasing sequence in $\mathcal{H} \mathcal{T}_{n}$ converging to 1 .

Proof of Corollary 1.6. - Fix an algebraically closed field $k$ of characteristic zero. Suppose that we have a strictly increasing sequence $\left\{c_{m}\right\}_{m}$ in $\mathcal{H} \mathcal{T}_{n}(k)$. By Corollary 3.8, we may write $c_{m}=\operatorname{lct}_{0}\left(f_{m}\right)$ for some $f_{m} \in k\left[x_{1}, \ldots, x_{n}\right]$ with $f_{m}(0)=0$. Theorem 1.3 (see also Remark 4.5) gives $c:=\lim _{m \rightarrow \infty} c_{m} \in \mathcal{H} \mathcal{T}_{n}(k)$. In particular $c \in \mathbf{Q}$, hence we may write $c=\frac{a}{b}$ for positive integers $a$ and $b$. Note that $a \leq b$, and let $N=n+b-a$ and

$$
g_{m}=f_{m}+\sum_{i=1}^{b-a} y_{i}^{b} \in k\left[x_{1}, \ldots, x_{n}, y_{1}, \ldots, y_{b-a}\right] .
$$

A special case of the Thom-Sebastiani Theorem (see Proposition 8.21 in [11]) implies that

$$
\operatorname{lct}_{0}\left(g_{m}\right)=\min \left\{1, \operatorname{lct}_{0}\left(f_{m}\right)+\sum_{i=1}^{b-a} \frac{1}{b}\right\}=\operatorname{lct}_{0}\left(f_{m}\right)+\frac{b-a}{b},
$$

hence $\left\{\operatorname{lct}\left(g_{m}\right)\right\}_{m}$ is a strictly increasing sequence in $\mathcal{H} \mathcal{T}_{N}(k)$ converging to 1 .

We now turn to the proof of Proposition 1.7 from the introduction. In fact, we will give a stronger statement in Proposition 5.2 below, that also interprets the ACC Conjecture as a semicontinuity property of log canonical thresholds of formal power series.

From now on we fix an uncountable algebraically closed field $k$ of characteristic zero. We consider the set $R=k \llbracket x_{1}, \ldots, x_{n} \rrbracket$ as the set of $k$-valued points of an infinite-dimensional affine space over $k$, parametrizing the coefficients of the power series. As such, it carries a natural Zariski topology.

This can be described, more precisely, as follows. If $\mathfrak{m}$ denotes the maximal ideal of $R$, then $R \simeq \operatorname{proj} \lim _{\ell} R / \mathfrak{m}^{\ell}$. Each $R / \mathfrak{m}^{\ell}$ parametrizes polynomials over $k$ of degree $<\ell$, and as such it consists of the $k$-points of an affine space of dimension $\left(\begin{array}{c}n+\ell-1 \\ n\end{array}\right)$. The Zariski topology on $R$ is the projective limit of the Zariski topologies on each of the $R / \mathfrak{m}^{\ell}$. Note that $\mathfrak{m} \subset R$ is a closed subset.

We denote by $\psi_{\ell}: R \rightarrow R / \mathfrak{m}^{\ell}$ the natural projection maps. A subset of $R$ is a cylinder if it is of the form $\psi_{\ell}^{-1}(S)$ for some $\ell$ and some subset $S$ of $R / \mathfrak{m}^{\ell}$ (note that we do not put any condition on $S$ ). A cylinder $C$ is constructible, open or closed if $S$ is constructible, open or closed, respectively. Since the projections $R / \mathfrak{m}^{\ell+1} \rightarrow R / \mathfrak{m}^{\ell}$ are continuous and open (being flat), it follows that these notions are well-defined. These are variants of the corresponding notions when instead of $R$ one considers the space of arcs of a smooth variety (see for example [7]).

REMARK 5.1. - A variant of an argument due to Batyrev [2] in the case of spaces of arcs (see also Lemma 1.2 in [7]) implies that if $C_{1} \supseteq C_{2} \supseteq \cdots$ is a sequence of constructible cylinders with $\cap_{m} C_{m}=\varnothing$, then $C_{m}=\varnothing$ for some $m$. This is the key point where we use the fact that $k$ is uncountable. 
For every $n \geq 1$ and $c \in \mathbf{R}$, we consider the set

$$
\mathcal{R}_{n}(c):=\{f \in \mathfrak{m} \mid \operatorname{lct}(f) \geq c\} .
$$

Proposition 5.2. - For every $n \geq 1$ and $c \in \mathbf{R}$, the following assertions are equivalent:

i) $c$ is not a point of accumulation from below of $\mathcal{H} \mathcal{T}_{n}$.

ii) $\mathcal{R}_{n}(c)$ is a cylinder in $R$.

iii) $\mathcal{R}_{n}(c)$ is open in $\mathfrak{m}$.

Proof. - For every $f \in R$ and every nonnegative integer $d$, we denote by $f_{\leq d}$ the truncation of $f$ of degree $\leq d$. It is convenient to consider also the map $\iota_{d}: R / \mathfrak{m}^{d+1} \rightarrow R$ that identifies each coset $h+\mathfrak{m}^{d+1}$ with the corresponding polynomial $h_{\leq d}$ of degree $\leq d$.

In order to prove that i) $\Rightarrow$ ii) note that if $c$ is not a point of accumulation from below for the set $\mathcal{H} \mathcal{T}_{n}$, then there is $\varepsilon>0$ such that $(c-\varepsilon, c) \cap \mathcal{H} \mathcal{T}_{n}=\varnothing$. It follows from Corollary 2.10 that if $\frac{n}{d+1}<\frac{\varepsilon}{2}$, then $f \in \mathcal{R}_{n}(c)$ if and only if $\operatorname{lct}_{0}\left(f_{\leq d}\right)>c-\frac{\varepsilon}{2}$. This implies that the condition for having $f \in \mathcal{R}_{n}(c)$ depends only on $\psi_{d+1}(f)$. Therefore $\mathcal{R}_{n}(c)=\psi_{d+1}^{-1}\left(\psi_{d+1}\left(\mathcal{R}_{n}(c)\right)\right)$, hence it is a cylinder.

Conversely, suppose now that we have a strictly increasing sequence $c_{m}$ in $\mathcal{H} \mathcal{T}_{n}$ converging to $c$, and that $\mathcal{R}_{n}(c)$ is a cylinder. We note that in general, for every $c^{\prime}>0$, we may write $\mathcal{R}_{n}\left(c^{\prime}\right)$ as a countable intersection of constructible cylinders. Indeed, it follows from Corollary 2.10 that we can write

$$
\mathcal{R}_{n}\left(c^{\prime}\right)=\bigcap_{\ell \geq 1} \psi_{\ell}^{-1}\left(S_{\ell}\right)
$$

where $S_{\ell}:=\left\{h+\mathfrak{m}^{\ell} \in \mathfrak{m} / \mathfrak{m}^{\ell} \mid \operatorname{lct}_{0}\left(h_{\leq \ell-1}\right) \geq c^{\prime}-\frac{n}{\ell}\right\}$ is open in $\mathfrak{m} / \mathfrak{m}^{\ell}$ by the Semicontinuity Theorem for log canonical thresholds (see Corollary 9.5.39 in [13]).

Therefore we write

$$
\mathcal{R}_{n}\left(c_{m}\right)=\bigcap_{\ell \geq 1} C_{\ell}^{m}
$$

where each $C_{\ell}^{m}$ is a constructible cylinder. Note that $\sup _{m} c_{m}=c$, hence

$$
\mathcal{R}_{n}(c)=\bigcap_{m} \mathcal{R}_{n}\left(c_{m}\right)=\bigcap_{m, \ell} C_{\ell}^{m} .
$$

Since $\mathcal{R}_{n}(c)$ is a cylinder, it follows from Remark 5.1 that $\mathcal{R}_{n}(c)$ is the intersection of finitely many $C_{\ell}^{m}$. In particular, $\mathcal{R}_{n}(c)=\mathcal{R}_{n}\left(c_{m}\right)$ for $m \gg 0$. However, by Corollary 3.8 we can find $h_{m} \in R$ such that $c_{m}=\operatorname{lct}\left(h_{m}\right)$. Since $c_{m}<c$ for every $c$, we have $h_{m} \in \mathcal{R}_{n}\left(c_{m}\right) \backslash \mathcal{R}_{n}(c)$ for all $m$, a contradiction. This completes the proof of i) $\Leftrightarrow$ ii).

Suppose now that $c$ is fixed, and that $\mathcal{R}_{n}(c)$ is a cylinder. In this case, we can write $\mathcal{R}_{n}(c)=\psi_{\ell}^{-1}(S)$, and $S$ can be identified via $\iota_{\ell-1}$ with the set of those polynomials $g$ of degree $\leq \ell-1$ such that $g(0)=0$ and $\operatorname{lct}_{0}(g) \geq c$. The Semicontinuity Theorem for $\log$ canonical thresholds implies that $S$ is open in $\mathfrak{m} / \mathfrak{m}^{\ell}$, hence $\mathcal{R}_{n}(c)$ is open in $\mathfrak{m}$. This shows that ii) $\Rightarrow$ iii).

Conversely, suppose that $\mathcal{R}_{n}(c)$ is open in $\mathfrak{m}$. Since $\mathfrak{m} \backslash \mathcal{R}_{n}(c)$ is closed, it follows from the definition of the Zariski topology on $R$ that we can write

$$
\mathfrak{m} \backslash \mathcal{R}_{n}(c)=\bigcap_{\ell \geq 1} \psi_{\ell}^{-1}\left(Z_{\ell}\right)
$$


for suitable closed subsets $Z_{\ell} \subseteq R / \mathfrak{m}^{\ell}$. We may clearly assume that $\psi_{\ell}^{-1}\left(Z_{\ell}\right) \supseteq \psi_{\ell+1}^{-1}\left(Z_{\ell+1}\right)$ for every $\ell$. On the other hand, we have seen that we can write

$$
\mathcal{R}_{n}(c)=\bigcap_{\ell \geq 1} C_{\ell}
$$

where each $C_{\ell}$ is a constructible cylinder, and we may assume that $C_{\ell} \supseteq C_{\ell+1}$ for every $\ell$. We deduce that if we put $C_{\ell}^{\prime}=C_{\ell} \cap \psi_{\ell}^{-1}\left(Z_{\ell}\right)$, then $C_{\ell}^{\prime} \supseteq C_{\ell+1}^{\prime}$ for every $\ell$, and $\bigcap_{\ell} C_{\ell}^{\prime}=\varnothing$. It follows from Remark 5.1 that there is $\ell$ such that $C_{\ell}^{\prime}=\varnothing$. In this case we have $C_{\ell}=\mathcal{R}_{n}(c)=$ $\mathfrak{m} \backslash \psi_{\ell}^{-1}\left(Z_{\ell}\right)$, hence $\mathcal{R}_{n}(c)$ is a cylinder. This completes the proof of the proposition.

Remark 5.3. - Suppose that Conjecture 1.1 holds for $n$, and let $c>0$. It follows from the above proposition that if $k$ is an uncountable algebraically closed field of characteristic zero, then we can find $N(n, c)$ such that the condition for $f \in k \llbracket x_{1}, \ldots, x_{n} \rrbracket$ with $f(0)=0$ to satisfy lct $(f) \geq c$ depends only on the truncation of $f$ up to level $N(n, c)$. In fact, this integer $N(n, c)$ is independent on $k$ : we have seen in the proof of the proposition that it depends only on the largest element in $\mathcal{H} \mathcal{T}_{n}$ that is $<c$. Moreover, $N(n, c)$ satisfies the same property for formal power series over every algebraically closed field $k$ of characteristic zero, as can be seen by taking an uncountable extension of $k$.

Remark 5.4. - We deduce from Corollary 1.4 that in order to prove Conjecture 1.1 for a given $n$ it is enough to show that every $\mathcal{R}_{n}(c)$ is a cylinder, for $c \in \mathbf{Q}$. Furthermore, it follows from Corollary 1.6 that in order to prove Conjecture 1.1, it is enough to show that for every $n$ the set $\mathcal{R}_{n}(1)$ is a cylinder.

\section{REFERENCES}

[1] V. Alexeev, Two two-dimensional terminations, Duke Math. J. 69 (1993), 527-545.

[2] V. V. Batyrev, Stringy Hodge numbers of varieties with Gorenstein canonical singularities, in Integrable systems and algebraic geometry (Kobe/Kyoto, 1997), World Sci. Publ., River Edge, NJ, 1998, 1-32.

[3] C. BIRKAR, Ascending chain condition for log canonical thresholds and termination of log flips, Duke Math. J. 136 (2007), 173-180.

[4] C. Birkar, P. Cascini, C. D. Hacon, J. M'Kernan, Existence of minimal models for varieties of general type, preprint arXiv:math.AG/0610203.

[5] M. Blickle, M. Mustață, K. E. Smith, F-thresholds of hypersurfaces, to appear in Trans. Amer. Math. Soc., available at arXiv:math/0705.1210.

[6] L. van den Dries, K. Schmidt, Bounds in the theory of polynomial rings over fields. A nonstandard approach, Invent. Math. 76 (1984), 77-91.

[7] L. Ein, R. Lazarsfeld, M. MustațǍ, Contact loci in arc spaces, Compos. Math 140 (2004), 1229-1244.

[8] L. Ein, R. Lazarsfeld, K. E. Smith, D. Varolin, Jumping coefficients of multiplier ideals, Duke Math. J. 123 (2004), 469-506.

[9] L. Ein, M. Mustaţă, Jet schemes and singularities, to appear in Proceedings of the 2005 AMS Summer Research Institute in Algebraic Geometry, available at arXiv:math.AG/0612862. 
[10] R. Goldblatt, Lectures on the hyperreals. An introduction to nonstandard analysis, Graduate Texts in Math. 188, Springer, 1998.

[11] J. Kollár, Singularities of pairs, in Algebraic geometry - Santa Cruz 1995, Proc. Sympos. Pure Math. 62, Amer. Math. Soc., 1997, 221-287.

[12] J. Kollár et al., Flips and abundance for algebraic threefolds. A summer seminar at the University of Utah (Salt Lake City, 1991), Astérisque 211 (1992), 1-258.

[13] R. Lazarsfeld, Positivity in algebraic geometry. II, Ergebnisse Math. Grenzg. 49, Springer, 2004.

[14] H. Matsumura, Commutative ring theory, second ed., Cambridge Studies in Advanced Mathematics 8, Cambridge University Press, 1989.

[15] J. M ${ }^{\mathrm{C}}$ Kernan, Y. Prokhorov, Threefold thresholds, Manuscripta Math. 114 (2004), 281-304.

[16] M. Mustaţă, Singularities of pairs via jet schemes, J. Amer. Math. Soc. 15(2002), 599615.

[17] H. Schoutens, Bounds in cohomology, Israel J. Math. 116 (2000), 125-169.

[18] V. V. Shokurov, Three-dimensional log perestroikas, Izv. Ross. Akad. Nauk Ser. Mat. 56 (1992), 105-203.

[19] M. Temkin, Desingularization of quasi-excellent schemes in characteristic zero, $A d v$. Math. 219 (2008), 488-522.

\author{
Tommaso de Fernex \\ Department of Mathematics \\ University of Utah \\ Salt Lake City, UT 48112, USA \\ E-mail: defernex@math.utah.edu \\ Mircea MustațĂ \\ Department of Mathematics \\ University of Michigan \\ Ann Arbor, MI 48109, USA \\ E-mail: mmustata@umich.edu
}

